TITLE:

\title{
Different cortical projections from three subdivisions of the rat lateral posterior thalamic nucleus: a single-neuron tracing study with viral vectors.
}

$\operatorname{AUTHOR}(\mathrm{S})$ :

Nakamura, Hisashi; Hioki, Hiroyuki; Furuta, Takahiro; Kaneko, Takeshi

\section{CITATION:}

Nakamura, Hisashi ...[et al]. Different cortical projections from three subdivisions of the rat lateral posterior thalamic nucleus: a single-neuron tracing study with viral vectors.. The European journal of neuroscience 2015, 41(10): 1294-1310

\section{ISSUE DATE:}

2015-04-02

URL:

http://hdl.handle.net/2433/201991

\section{RIGHT:}

This is the peer reviewed version of the following article: Nakamura, H., Hioki, H., Furuta, T. and Kaneko, T. (2015), Different cortical projections from three subdivisions of the rat lateral posterior thalamic nucleus: a single-neuron tracing study with viral vectors. European Journal of Neuroscience, 41: 1294-1310, which has been published in final form at http://dx.doi.org/10.1111/ejn.12882. This article may be used for non-commercial purposes in accordance with Wiley Terms and Conditions for Self-Archiving.; The full-text file will be made open to the public on 2 April 2016 in accordance with publisher's 'Terms and Conditions for Self-Archiving':; この論文は出版社版でありません。引用の際に は出版社版をご確認ご利用ください。; This is not the published version. Please cite only the published version. 
Journal section: Neurosystems

Title

Different Cortical Projections from Three Subdivisions of the Rat Lateral Posterior

Thalamic Nucleus: A Single Neuron Tracing Study with Viral Vectors

Manuscript \# EJN-2014-12-22357

Abbreviated title: Projections of lateral posterior nucleus neuron

Hisashi Nakamura, Hiroyuki Hioki*, Takahiro Furuta and Takeshi Kaneko

Department of Morphological Brain Science, Graduate School of Medicine, Kyoto University, Kyoto 606-8501, Japan

*Correspondence to: Dr. Hiroyuki Hioki, M.D., Ph.D.

Department of Morphological Brain Science,

Graduate School of Medicine, Kyoto University,

Kyoto 606-8501, Japan

Phone: 81 (Japan)-75-753-4331, Fax: 81-75-753-4340

E-mail: hioki@mbs.med.kyoto-u.ac.jp

Number of pages: 48

Number of figures: 10

Number of tables: 3

Number of words

Whole manuscript: 9772 words

Abstract: 248 words

Introduction: 499 words

Keywords: axonal arborization, extrageniculate pathway, lateral posterior nucleus of the thalamus, Sindbis virus, single neuron labeling. 


\section{Abstract}

The lateral posterior thalamic nucleus (LP) is one of the components of the extrageniculate pathway in the rat visual system, and cytoarchitecturally divided into three subdivisions, lateral (LPl), rostromedial (LPrm) and caudomedial portions (LPcm). To clarify the differences in the dendritic fields and axonal arborizations among the three subdivisions, we applied a single neuron labeling technique with viral vectors to LP neurons. The proximal dendrites of LP1 neurons were more numerous than those of LPrm and LPcm neurons, and LPrm neurons tended to have wider dendritic fields than those of LPl neurons. We then analyzed the axonal arborizations of LP neurons by reconstructing the axon fibers in the cortex. LPl, LPrm and LPcm were different from one another in terms of the projection targets: the main target cortical regions of LPl and LPrm neurons were the secondary and primary visual areas, whereas those of LPcm neurons were the postrhinal and temporal association areas. Furthermore, the principal target cortical layers of LPl neurons in the visual areas were middle layers, but that of LPrm neurons was layer 1. This indicates that LPl and LPrm neurons can be categorized into the core- and matrix-types of thalamic neurons in the visual areas, respectively. In addition, LPl neurons formed multiple axonal clusters within the visual areas, whereas the fibers of LPrm neurons were widely and diffusely distributed. It is therefore presumed that these two types of neurons play different roles in visual information processing by dual thalamocortical innervation of the visual areas. 


\section{Introduction}

The lateral posterior nucleus (LP) and pulvinar complex of the thalamic nuclei are components of the extrageniculate pathway, whereas the dorsal lateral geniculate nucleus (LGd) is the central relay station of the geniculo-striate pathway in the mammalian visual system (Schneider, 1969; Tohmi et al., 2014; for review, see Diamond \& Hall, 1969; Diamond, 1976). The LP-pulvinar complex not only receives direct retinal inputs, but also has the inputs from the superficial layers of the superior colliculus and pretectal visual regions (for review, see Jones, 2007). The LP and pulvinar are distinct thalamic nuclei in many mammals including carnivores and primates, but the LP has been identified as a sole element of the LP-pulvinar complex in the rat brain (for review, see Jones, 2007).

The rat LP has been cytoarchitecturally divided into three subdivisions, rostromedial (LPrm), caudomedial (LPcm), and lateral portions (LPl; Takahashi, 1985). The LPcm mainly receives the inputs from the superficial layers of the superior colliculus, whereas the LPrm and LPl chiefly have the inputs from the visual cortex (Perry, 1980; Mason \& Groos, 1981; Takahashi, 1985; Bourassa \& Deschênes, 1995; Shi \& Davis, 2001; Masterson et al., 2009). In addition, the LP receives a small amount of direct retinal inputs (Goodman et al., 1973; Yamadori, 1977; Ahmed et al., 1995). LP neurons then send axon fibers predominantly to the primary visual area (V1), lateral region of the secondary visual area (V21) and medial portion of the V2 (V2m) (Hughes, 1977; Olavarria, 1979; Herkenham, 1980; Perry, 1980; Schobber, 
1981; Reep et al., 1994; Shi \& Davis, 2001).

It has been reported that the target cortical layers of LP neurons are layer (L) 1, L3 and L4 in the rat V2, and L1 and L5 in the V1 (Hughes, 1977; Herkenham, 1980; Parnavelas et al., 1981; Schobber, 1981; Rubio-Garrido et al., 2009). Recently, the axonal arborizations of two single neurons in the LP were analyzed by using a juxtacellular labeling technique (Noseda et al., 2010, 2011). Since these two LP neurons mainly sent axon fibers to L3 and L4 but not to L1 in the V2, these neurons are considered to belong to core-type neurons in the recently proposed classification (for review, see Jones, 1998, 2001), where thalamic neurons are categorized into the core- and matrix-types based on the cortical projections (Rubio-Garrido et al., 2007, 2009; for review, see Clascá et al., 2012).

We have developed Sindbis virus vectors (Furuta et al., 2001; Nishino et al., 2008), which strongly express reporter protein under the powerful viral subgenomic promoter, and which are considered suitable for visualizing entire structures of a few or single neurons. Using the vectors, we have clearly demonstrated the axonal arborizations of single mesencephalic dopamine neurons (Matsuda et al., 2009) and thalamic neurons (Kuramoto et al., 2009, 2015; Ohno et al., 2012). In the present study, we applied this technique to reveal the axonal arborizations of single LP neurons, and demonstrated that LP neurons in each subdivision had different characteristics in the cortical target areas and layers. 


\section{Materials and Methods}

\section{Injection of viral vector or anterograde tracer and fixation of the brain}

All animal care and use were in accordance with the National Institutes of Health Guide for the Care and Use of Laboratory Animals, and the experiments were approved by the Committee for Animal Care and Use (MedKyo 13012) and that for Recombinant DNA Study (120093) in Kyoto University. Adult male Long-Evans and Wistar rats (250-350 g; Japan SLC, Hamamatsu, Japan) were used in the present study, and all efforts were made to minimize the suffering and number of animals used in the present study.

Forty-one Long-Evans rats and 26 Wistar rats were anesthetized by intraperitoneal injection of chloral hydrate $(35 \mathrm{mg} / 100 \mathrm{~g}$ body weight). For single neuron labeling, we utilized Sindbis virus vector, which expresses palmitoylation (pal) site-attached green fluorescent protein (palGFP; Furuta et al., 2001) or monomeric red fluorescent protein (pal-mRFP; Nishino et al., 2008). Palmitoylated fluorescent protein, palGFP or pal-mRFP, was targeted to the plasma membrane of the infected neurons (Moriyoshi et al., 1996), and effective in visualizing whole structures of neurons. We injected a mixture of Sindbis virus vectors into the LP of the rats bilaterally $(3.5-5.4 \mathrm{~mm}$ posterior to the bregma, $1.7-3.0 \mathrm{~mm}$ lateral to the midline, and $4.0-4.5 \mathrm{~mm}$ deep from the brain surface) by pressure through a glass micropipette attached to a Picospritzer II (General Valve Corporation, East Hanover, NJ). The mixture contained Sindbis viral vectors expressing palGFP $\left(3-6 \times 10^{4}\right.$ infectious 
units (IU); Furuta et al., 2001) and pal-mRFP (3-6 $610^{4}$ IU; Nishino et al., 2008) in $0.2 \mu \mathrm{L}$ of $5 \mathrm{mM}$ sodium phosphate ( $\mathrm{pH} 7.4)$-buffered $0.9 \%(\mathrm{w} / \mathrm{v})$ saline (PBS) containing 2\% (w/v) bovine serum albumin (BSA). For conventional anterograde tracing experiments, 11 Long-Evans rats were anesthetized as described above, and injected with $2.5 \%(\mathrm{w} / \mathrm{v})$ Phaseolus vulgaris leucoagglutinin (PHA-L; 300105; JOIL MILLS, Tokyo, Japan) in $0.1 \mathrm{M}$ sodium phosphate buffer (PB; pH7.4) iontophoretically by passing 7-s long, $2-\mu \mathrm{A}$ positive current pulses at 7 -s intervals for 30 min through a glass micropipette. The rats were allowed to survive for $48-54 \mathrm{~h}$ or 14 days after viral vectors or PHA-L injection, respectively.

The rats injected with viral vectors or PHA-L and 3 control Long-Evans rats were anesthetized deeply with chloral hydrate $(70 \mathrm{mg} / 100 \mathrm{~g})$, and perfused transcardially with 300 $\mathrm{mL}$ of PBS, followed by $300 \mathrm{~mL}$ of $3 \%(\mathrm{v} / \mathrm{v})$ formaldehyde, $75 \%(\mathrm{v} / \mathrm{v})$-saturated picric acid (0.95\% (w/v) picric acid), and $0.1 \mathrm{M} \mathrm{Na}_{2} \mathrm{HPO}_{4}$ (adjusted with $\mathrm{NaOH}$ to $\mathrm{pH} 7.2$ ). The brains were then removed and postfixed for $4 \mathrm{~h}$ at room temperature with the same fixative. After cryoprotection with $30 \%(\mathrm{w} / \mathrm{v})$ sucrose in $0.1 \mathrm{M} \mathrm{PB}$, the brains were cut into $50-\mu \mathrm{m}$-thick coronal or sagittal sections on a freezing microtome, and the sections were collected serially in $0.1 \mathrm{M} \mathrm{PB}$. 
Double immunofluorescence labeling for calbindin D-28k and vesicular glutamate

\section{transporter 2}

All the following incubations were performed at room temperature and followed by a rinse with PBS containing $0.3 \%(\mathrm{v} / \mathrm{v})$ Triton X-100 (PBS-X) for 10 min twice. The sections of control rats were incubated overnight with 1/1000-diluted mouse monoclonal IgG1 to calbindin D-28k (CB; C9848; Sigma-Aldrich, St Louis, MO) and $1 \mu \mathrm{g} / \mathrm{mL}$ affinity-purified rabbit antibody to vesicular glutamate transporter 2 (VGluT2; Hioki et al., 2003) in PBS-X containing $1 \% \quad(\mathrm{v} / \mathrm{v})$ goat serum and $0.12 \% \quad(\mathrm{w} / \mathrm{v}) \quad \lambda$-carrageenan (PBS-XCG). Lambda-carrageenan makes the incubation medium highly viscous and contributes to avoiding uneven binding of antibodies to sections (Hioki et al., 2013). The sections were subsequently incubated for $2 \mathrm{~h}$ with $5 \mu \mathrm{g} / \mathrm{mL}$ Alexa Fluor (AF) 647-conjugated goat antibody to mouse IgG (A21236; Life Technologies, Carlsbad, CA) and AF488-conjugated goat antibody to rabbit IgG (A11034; Life Technologies) in PBS-XCG and then for 30 min with 1/200-diluted NeuroTrace 530/615 red fluorescent Nissl stain (N21482; Life Technologies) in PBS-X. The sections were mounted onto gelatinized glass slides, coverslipped with $90 \%$ (v/v) glycerol and $2.5 \%(\mathrm{w} / \mathrm{v})$ triethylenediamine in $20 \mathrm{mM}$ Tris- $\mathrm{HCl}(\mathrm{pH} 7.6)$, and then observed under a TCS SP8 confocal laser-scanning microscope (Leica Microsystems, Wetzlar, Germany) with a $\times 5$ objective lens (HCX PL FLUOTAR; NA $=0.15$; Leica) and HyD detector (Leica). AF488, NeuroTrace red or AF647 was excited with 488, 552 or $638 \mathrm{~nm}$ 
laser beam and observed through 510-540, 610-635 or 665-800 nm emission prism window, respectively. All confocal images were arranged and modified $(<40 \%$ contrast enhancement) in software Canvas 12 (ACD Systems International Inc., Victoria, Canada) and saved as 8-bit/color TIFF files.

\section{Characterization of palGFP-or pal-mRFP-expressing thalamic neurons}

The sections including the injection site of viral vectors were observed under an Axioplan 2 epifluorescence microscope (Carl Zeiss, Oberkochen, Germany) with a filter set for GFP (excitation filter, 450-490 nm; emission, 515-565 nm) or mRFP (excitation, 530-585 nm; emission, $\geq 615 \mathrm{~nm}$ ) to detect thalamic neurons infected with the virus expressing palGFP or pal-mRFP. When infected neurons were found, the sections containing palGFP- or pal-mRFP-positive neurons were incubated overnight with 1/1000-diluted anti-CB mouse monoclonal IgG1 in PBS-XCG, and then for $2 \mathrm{~h}$ with $5 \mu \mathrm{g} / \mathrm{mL}$ AF647-conjugated goat antibody to mouse IgG in PBS-XCG. The sections were further incubated $30 \mathrm{~min}$ with 1/200-diluted NeuroTrace 530/615 red fluorescent Nissl stain or NeuroTrace 500/525 green fluorescent Nissl stain (N21480; Life Technologies) in PBS-X. Subsequently, fluorescence images were acquired by a TCS SP8 confocal laser-scanning microscope, using a $\times 5$ or $\times 40$ objective lens (HCX PL FLUOTAR; NA = 0.6; Leica) at the pinhole of 4.2 or 1.0 Airy unit (optical slice thickness of 179.8 or $3.2 \mu \mathrm{m}$ ). GFP and NeuroTrace green, mRFP and 
NeuroTrace red, or AF647 were excited with 488, 552 or $638 \mathrm{~nm}$ laser beam and observed through $510-540,580-630$ or $665-800 \mathrm{~nm}$ emission prism window, respectively. The location of palGFP- or pal-mRFP-labeled neurons was examined in reference to Nissl-like staining and CB immunoreactivity.

\section{Immunoperoxidase staining}

GFP or mRFP immunoreactivity was visualized by combining the avidin-biotinylated peroxidase complex $(\mathrm{ABC})$ method with the biotinylated tyramine (BT)-glucose oxidase (GO) amplification (Furuta et al., 2009; Kuramoto et al., 2009). Briefly, all the serial sections were incubated overnight with $0.4 \mu \mathrm{g} / \mathrm{mL}$ affinity-purified rabbit antibody to GFP (Tamamaki et al., 2000) or $0.2 \mu \mathrm{g} / \mathrm{mL}$ affinity-purified rabbit antibody to mRFP (Hioki et al., 2010), respectively, in PBS-XCG. The sections were subsequently incubated for $1 \mathrm{~h}$ with 10 $\mu \mathrm{g} / \mathrm{mL}$ biotinylated goat antibody to rabbit IgG (BA-1000; Vector Laboratories, Burlingame, $\mathrm{CA})$ in PBS-XCG and then for $1 \mathrm{~h}$ with $\mathrm{ABC}$ (1:100; ABC-Elite; Vector Laboratories) in PBS-X. After a rinse in $0.1 \mathrm{M}$ PB for $10 \mathrm{~min}$ twice, the sections were incubated for $30 \mathrm{~min}$ in the BT-GO reaction mixture containing $2.55 \mu \mathrm{M}$ BT, $3 \mu \mathrm{g} / \mathrm{mL}$ of $\mathrm{GO}(257 \mathrm{U} / \mathrm{mg}$; 16831-14; nacalai tesque, Kyoto, Japan), $2 \mathrm{mg} / \mathrm{mL}$ of $\beta$-D-glucose (16804-32; nacalai tesque), and $2 \%(\mathrm{w} / \mathrm{v}) \mathrm{BSA}$ in $0.1 \mathrm{M} \mathrm{PB}$, followed by a wash with PBS-X for $10 \mathrm{~min}$ twice. The sections were again incubated for $1 \mathrm{~h}$ with $\mathrm{ABC}$ in PBS-X, and the bound peroxidase was 
finally developed brown by reaction for 30-60 min with $0.02 \%$ (w/v) diaminobenzidine-4 $\mathrm{HCl}(\mathrm{DAB})$ and $0.0001 \%(\mathrm{v} / \mathrm{v}) \mathrm{H}_{2} \mathrm{O}_{2}$ in $50 \mathrm{mM}$ Tris- $\mathrm{HCl}(\mathrm{pH}$ 7.6).

The sections obtained from PHA-L-injected rats were incubated overnight with 3.75 $\mu \mathrm{g} / \mathrm{mL}$ rabbit anti-PHA-L antibody (AL-1801-2; E-Y Laboratories, San Mateo, CA) in PBS-XCG and then for $1 \mathrm{~h}$ with $10 \mu \mathrm{g} / \mathrm{mL}$ biotinylated goat antibody to rabbit $\mathrm{IgG}$ in PBS-XCG, followed by incubation for $1 \mathrm{~h}$ with 1/100-dilulted ABC-Elite in PBS-X. Subsequently, the bound peroxidase was developed brown by reaction for 30-60 min with $0.02 \%(\mathrm{w} / \mathrm{v}) \mathrm{DAB}$ and $0.0005 \%(\mathrm{v} / \mathrm{v}) \mathrm{H}_{2} \mathrm{O}_{2}$ in $50 \mathrm{mM}$ Tris-HCl.

All the stained sections were serially mounted onto gelatinized glass slides, dried up, dehydrated in an ethanol series, cleared in xylene, and finally coverslipped. After reconstruction of palGFP-, pal-mRFP-, or PHA-L-labeled axon fibers as described below, the sections were counterstained for Nissl with $0.2 \%(\mathrm{w} / \mathrm{v})$ cresyl violet to identify the cytoarchitecture of the forebrain. Fine morphological indices, such as inter-varicosity intervals, were observed under a microscope (Optiphot; Nikon, Tokyo, Japan) with an oil-immersion $\times 100$ objective lens (PlanApo100; NA = 1.4; Nikon).

\section{Reconstruction and morphological analysis of single LP neurons}

The cell body and dendrites of stained LP neurons in coronal sections were reconstructed under a microscope (Optiphot) with a camera lucida using a $\times 40$ objective lens (PlanApo40; 
NA $=0.95$; Nikon). Those of LP neurons in sagittal sections were reconstructed three-dimensionally under a microscope (Vanox; Olympus, Tokyo, Japan) equipped with a Neurolucida system (MicroBrightField, Williston, VT) using a $\times 40$ objective lens (SPlanApo40; NA $=0.95$; Olympus), rotated, and projected to the coronal plane. The axonal arborizations were reconstructed as described previously (Ohno et al., 2012). Briefly, a whole coronal or sagittal section was automatically captured into a large color image with a spatial resolution of $1.038 \mu \mathrm{m} /$ pixel using a TOCO digital slide scanner (CLARO, Aomori, Japan) with a $\times 10$ objective lens (EC Plan-Neofluar; NA $=0.30$; Zeiss). On the images, we traced and digitized the axonal fibers with a pen tablet (Bamboo Tablet; Wacom, Saitama, Japan) and software Canvas 12. The axonal fibers were thereby reconstructed two-dimensionally section by section onto the coronal or sagittal plane, and the digitized fibers from all the sections were superimposed in the computer. The length of varicose axon fibers was calculated by using Adobe Illustrator CS4 (Adobe Systems Inc., San Jose, CA).

The axon varicosity was defined by the localized swelling of axon fibers with $\geq 1$-5-fold larger diameters than the thickness of inter-varicosity axon fibers, based on the previous reports (Kuramoto et al., 2009, 2015; Tanaka et al., 2011; Ohno et al., 2012). One hundred varicose axon fibers, which ran parallel to the section surface, were randomly selected in each layer within each area from the sections of the PHA-L tracing study, and the mean inter-varicosity interval was calculated by using software Canvas 12 (Supplementary 
Table 1).

\section{Cytoarchitecture of cortical areas}

Coronal sections of control rats were serially mounted onto gelatinized glass slides, dried up, dehydrated in an ethanol series, cleared in xylene, stained for Nissl with $0.2 \%$ (w/v) cresyl violet, and finally coverslipped. Cortical areas and layers were determined by the Nissl-stained sections and rat brain atlases (Zilles, 1985; Swanson, 2004; Paxinos \& Watson, 2007).

\section{Statistical analysis}

The cortical projection or dendritic morphology of LPl, LPrm, and LPcm neurons was compared by one- or two-way analysis of variance (ANOVA) followed by Tukey's or Bonferroni post hoc multiple comparison test by using GraphPad Prism 4 (GraphPad Software Inc., San Diego, CA). 


\section{Results}

\section{Cyto- and chemoarchitecture of the LP}

The LP has been cytoarchitecturally divided into the LP1, LPrm, and LPcm (Fig. 1A1, B1, C1, D1) according to Takahashi (1985). Although the LP1 is further classified into rostral and caudal portions by Paxinos and Watson (2007), the border was unclear in the present study with Cresyl violet and Nissl-like fluorescent staining, as previously described (Takahashi, 1985). The LPcm was clearly distinguished from the LPl and LPrm by a dense distribution of VGluT2-immunoreactive axon terminals (Fig. 1A2, B2, C2, D2), which were derived mainly from the superior colliculus (Masterson et al., 2009). We revealed that the region also showed intense immunoreactivity for $\mathrm{CB}$ (Fig. 1B3, C3, D3), and thus the immunoreactivity should be helpful as a chemical marker for the LPcm. In addition, we observed weak to moderate immunoreactivity for CB in the LPl and LPrm, and almost no immunoreactivity in the pretectal regions and LGd (Fig. 1A3, B3, C3, D3). This finding indicates that $\mathrm{CB}$ immunoreactivity is also useful for demarcating the border between the LP and pretectal regions or LGd. In the present study, we divided the LP into three portions on the basis of Nissl-stain-like cytoarchitecture with the aid of CB immunoreactivity. Furthermore, we examined whether or not the virus infected neurons showed the immunoreactivity for $\mathrm{CB}$ as one of the markers for matrix-type thalamic neurons. It should be noticed that the immunoreactivity for parvalbumin (PV), one of the markers for core-type 
thalamic neurons, was not found in the rodent thalamic nuclei except the reticular nucleus (for review, see Jones, 1998).

\section{Selection of single LP neurons for the morphological analysis}

A mixture of palGFP- and pal-mRFP-expressing Sindbis virus vectors was injected at an adequate dilution into both hemispheres of 67 rat brains (134 hemispheres). Thirty-one hemispheres containing only one palGFP- or pal-mRFP-labeled neuron in the thalamus were selected under an epifluorescence microscope (arrowheads in Fig. 2A-C) and further processed. We performed fluorescent Nissl-like staining (Fig. 2A) and immunofluorescence staining for CB (Fig. 2B), and found that 8,6 and 6 labeled neurons were located in the LPl, LPrm and LPcm, respectively. Most of the cell bodies, except LP1 neuron \#4, were immunoreactive for CB (Fig. 2C') both in the CB-weak (LPl and LPrm) and CB-intense regions (LPcm). Subsequently, the sections were processed for immunoperoxidase staining for GFP or mRFP to completely visualize the whole structures of single neurons (Fig. 2D-H). The axon fibers of $7 \mathrm{LPl}, 4 \mathrm{LPrm}$ and $5 \mathrm{LPcm}$ neurons were clearly labeled, and always formed a small varicosity at the end of the fibers (Kuramoto et al., 2009, 2015; Ohno et al., 2012). These neurons were reconstructed and analyzed in the present study, and each neuron was numbered from lateral to medial positions within the respective subdivisions of the LP (Fig. 3A-D). The remaining $1 \mathrm{LPl}, 2 \mathrm{LPrm}$ and $1 \mathrm{LPcm}$ neurons were excluded 
from the present analysis, since we observed that their axonal labeling faded away at the distal portion of their axonal arborizations. As far as observed in the present study, we found no sign for the modification of axon fibers, such as growth cone-like swelling of axon terminals, as reported in our previous single neuron studies using palGFP and pal-mRFP Sindbis virus vectors (Kuramoto et al., 2009, 2015; Matsuda et al., 2009; Fujiyama et al., 2011; Ohno et al., 2012; Koshimizu et al., 2013).

All the 16 LP neurons extended multipolar dendrites (Fig. 2D), and the reconstructed dendrites were projected onto the coronal plane (Fig. 3E). The dendritic fields of LP1 neurons \#1-7 were denser than those of LPrm neurons \#8-11 and of LPcm neurons \#12-16. By using Sholl analysis (Fig. 3F; Sholl, 1953), we determined that the dendrites of LP1 neurons were more numerous at a distance of 40-60 $\mu \mathrm{m}$ away from the cell body than those of LPrm and LPcm neurons ( $<<0.01$ and $\mathrm{p}<0.05)$. LPrm neurons tended to have wider dendritic arborizations than LPl neurons showing significant differences $(\mathrm{p}<0.01)$ at a distance of 140-160 $\mu \mathrm{m}$ away from the cell body. No significant difference was found in the dendritic fields between Long-Evans (LP neurons \#1, 4, 5, 6, 7, 9, 10, 12, 13, 16) and Wistar rats (LP neurons \#2, 3, 8, 11, 14, 15) by Sholl analysis. In addition, the dendrites of LP neurons spread radially and showed no orientation preference. Some dendrites were not actually confined to the subdivisions where the cell bodies were located (LP neurons \#6, 7, 9, 14). 


\section{Axon collaterals of LP neurons in the subcortical regions}

All the LP neurons reconstructed in the present study emitted axon collaterals to the thalamic reticular nucleus when their main axons left the thalamic region (Fig. $2 \mathrm{G}$ ) as reported in the previous single neuron labeling studies of thalamic neurons (Kuramoto et al., 2009, 2015; Ohno et al., 2012). Different from the previous findings, 4 of 16 LP neurons (2 LPl, 1 LPrm and 1 LPcm neurons) had short axon collaterals with varicosities inside of the LP (Supplementary Fig. 1), before emitting the collaterals to the thalamic reticular nucleus.

In the neostriatum, 4 of $7 \mathrm{LPl}$ neurons, 3 of $4 \mathrm{LPrm}$ neurons, and 4 of $5 \mathrm{LPcm}$ neurons emitted axon collaterals (Table 1, Fig. $2 \mathrm{H}$ ). The striatal projections from LPl and LPrm neurons were observed mainly in the dorsomedial to dorsal periphery of the neostriatum (Fig. 4G, 5A, G, Supplementary Fig. 2A, I, 3A, 4D), whereas those from LPcm neurons were found chiefly in the caudodorsal to caudolateral region (Fig. 6A, C, Supplementary Fig. 5A, G). It has been reported that LP neurons project to the dorsal portion of the neostriatum by using anterograde tract-tracing techniques (Funaki et al., 1998; Kamishina et al., 2008). Although not all the LP neurons examined in the present study sent axon fibers to the neostriatum, the present findings are in good agreement with those previous reports. In addition, the dorsal part of the neostriatum also receives the inputs from the visual cortex (Serizawa et al., 1994), suggesting that visual information from the visual cortex 
and LP may be integrated in the neostriatum.

\section{Cortical projections of LP neurons}

The main cortical target areas of LP1 neurons were the V2 (Fig 2E, F), followed by the V1 and retrosplenial area (Table 1, Fig. 4, Supplementary Fig. 2, 3). As shown in Figure 4A-F and Supplementary Figure 2A-E and F-H, two axonal clusters of LPl neurons \#2-4 were found in the two different regions in the V2, V2l and V2m. LP1 neurons \#3 and \#4 further formed an additional cluster in the V1. In the case of LP1 neurons \#1 and \#5, two or more axonal clusters were observed in the V21 (Fig. 4G-J, Supplementary Fig. 2I-L). Thus, 5 of 7 LP1 neurons formed 2-3 axonal clusters in the V2 (Fig. 4B, C, H-J, Supplementary Fig. 2B, E, G, H, J, K). The remaining two LPl neurons \#6 and \#7 formed clusters both in the V1 and V2 (Supplementary Fig. 3B, E, F).

LPrm neurons also sent varicose axon fibers mainly to the visual areas, especially to the V2. The fibers of LPrm neurons \#8-10 were more widely and diffusely distributed in the cortical regions than those of LP1 neurons (compare Fig. 5 and Supplementary Fig. 4 with Fig. 4 and Supplementary Fig. 2 and 3). In addition to the visual areas, LPrm neurons sent considerable varicose axon fibers to the temporal association, postrhinal, secondary motor and anterior cingulate areas (Table 1).

In contrast, the main target areas of 5 reconstructed LPcm neurons were not the 
visual areas, but the 'extravisual' areas (Table 1), such as the postrhinal and temporal association areas (Fig. 6A, C, Supplementary Fig. 5A, C, G). The cortical axon fibers of LPcm neurons formed a large axonal cluster in the postrhinal area and a much smaller or sparser cluster in the other cortical regions including the visual areas (Fig. 6B, D-G, Supplementary Fig. 5B, D-F, H, I).

We also noticed the difference in layer preference among the three subdivisions of the LP. LP1 neurons sent 7.0-27.2\% (15.3 \pm 7.5 ; mean \pm S.D. $)$ of varicose axon fibers to L1 (Table 2, red axon fibers in Fig. 4 and in Supplementary Fig. 2, 3) and 72.3-91.3\% (83.0 \pm 6.9) to L2-5 (yellow, green and blue fibers in Fig. 4 and in Supplementary Fig. 2, 3). In the case of LPrm neurons, $27.0-77.9 \%$ (48.7 \pm 23.2$)$ of axon fibers were observed in L1 (Table 2, red axon fibers in Fig. 5 and in Supplementary Fig. 4), although the main target regions of LPrm neurons were the same visual areas as LPl neurons (Table 1). These results indicate that LP1 and LPrm neurons prefer the middle cortical layers (L2-5) and L1 mainly in the visual areas. In contrast, some LPcm neurons (\#13 and \#16) sent a large number of varicose axon fibers to L1 in the 'extravisual' areas $(7.1-48.2 \%(26.2 \pm 17.6)$ of axon fibers; Table 2, red axon fibers in Fig. 6 and in Supplementary Fig. 5), but the preference was not so strong as that of LPrm neurons. Furthermore, individual neurons in the three subdivisions exhibited a variety of projection patterns in the cortical target areas and layers within two species, Long-Evans and Wistar rats. Although pigmented Long-Evans rats are generally considered 
to be superior to albino Wistar rats in visual function, similar projection patterns in each subdivision, as described above, were found in both two lines in the present study (Table 1, 2).

\section{PHA-L injection into the LP}

To reveal the population-level characteristics of the cortical projections of LP neurons, we injected a conventional anterograde tracer, PHA-L, into the three subdivisions of the LP (Fig. 7). The results of PHA-L experiments were summarized in Table 3. When PHA-L was injected into the LP1 (PH1-6; Fig. 7A-C), labeled axons were densely distributed in L2-5 of the V2, and moderately in L1 of the V2 and in L1-5 of the V1 (Fig. 7E, F). The labeled axons were also observed in the other areas such as the postrhinal, temporal association, retrosplenial, and anterior cingulate areas (Fig. 7G). After the injection of PHA-L into the LPrm (PH11-15; Fig. 7A, B), labeled fibers were found widely and intensely in L1 of the V2, V1, temporal association, postrhinal, and anterior cingulate areas (Fig. 7H-J). When the injection was made into the border between the LPl and LPrm (PH7-10; Fig. 7A-C), the distribution of axonal fibers in the visual areas was a mixture of the LPI and LPrm injection cases (data not shown). Finally, following the injection of PHA-L into the LPcm (PH16-21; Fig. 7C, D), dense terminal labeling was found always in the postrhinal and temporal association areas, and the chief targets were not the visual areas (Fig. $7 \mathrm{~K}-\mathrm{M})$. The present 
anterograde tracing experiments with PHA-L covered all the cortical regions to which single LP neurons sent axon fibers (Table 3), and thus support the results of the single neuron labeling experiments of LP neurons.

\section{Estimated number of varicosities in cortical axons}

As axon varicosities are essential components for synaptic transmission as presynaptic sites, it is important to estimate the number of axon varicosities of LP neurons in each cortical area and layer. We calculated the number of axon varicosities by dividing the length of varicose axons of single-labeled LP neurons by the mean inter-varicosity interval. The mean interval was measured in the sections of PHA-L anterograde labeling in each cortical area and layer (Supplementary Table 1). In these sections, axon varicosities were counted when the size of the varicosities was more than 1.5-fold larger than the thickness of the intervaricose fibers.

In Figure 8, the estimated number of axon varicosities of 16 single-labeled LP neurons is presented. In cortical areas, LP1 neurons \#1-7 principally sent axon varicosities to the V2 and/or V1, whereas LPrm neurons \#8-11 formed varicosities not only in the visual areas but also in the other regions such as the postrhinal and temporal association areas (Fig.

8A, B). All the reconstructed LPcm neurons sent only a few axon varicosities to the V1 and V2 (Fig. 8A), but preferred the postrhinal and temporal association areas as target areas (Fig.

8B). In addition, as revealed by the red bars in Figure 8, LPrm neurons apparently preferred 
L1, whereas LP1 neurons favored the middle layers (L2-5) in the visual areas. These results clearly indicate the differences in thalamocortical projections among the three subdivisions of the LP.

In Figure 9, the differences in the number of thalamocortical axon varicosities were statistically compared among reconstructed LPl, LPrm and LPcm neurons. Although the total number of axon varicosities was similar among the three neuronal groups, the main target areas of the three subdivisions were significantly different (Fig. 9A). LPl and LPrm neurons principally projected to the V1 and V2, whereas LPcm neurons mainly sent axon varicosities to the postrhinal and temporal association areas. In all the cortical areas that LP neurons projected to, the axon varicosities of LPrm neurons were distributed in L1 more preferentially than those of LPl and LPcm neurons (Fig. 9B, C). In addition, when the analysis was focused on the visual areas, LPl neurons displayed a significant preference for L4 compared with LPrm and LPcm neurons, and for L2/3 compared with LPrm neurons (Fig. 9D). These results lead to the concept that the LP is not a homogeneous structure and not simply topographic in the thalamocortical projections. 


\section{Discussion}

In the present study, whole structures of single neurons in the LP were successfully visualized by Sindbis virus vector. We revealed that three subdivisions of the LP, LPl, LPrm and LPcm, were different from one another in terms of the projection targets (Fig. 10): 1) the main target cortical regions of LPl and LPrm neurons were the visual areas, whereas those of LPcm neurons were the postrhinal and/or temporal association areas; 2) the principal target cortical layers of LP1 neurons in the visual areas were middle layers, but that of LPrm neurons was L1; and 3) most LP1 neurons formed multiple (2-3) axonal clusters within the V2 or in the V2 and V1.

The rat V2 has been revealed to receive strong inputs from LP neurons by retrograde tract-tracing experiments (Olavarria, 1979; Perry, 1980; Sanderson et al., 1991; Reep et al., 1994) as well as by anterograde tracing studies (Hughes, 1977; Herkenham, 1980; Schobber, 1981; Shi \& Davis, 2001). Not only the PHA-L experiment but also the single neuron tracing study in the present study is in good agreement with those previous reports: LPl and LPrm neurons projected mainly to the V2, whereas LPcm neurons sent axons mainly to the 'extravisual' areas such as the postrhinal and temporal association areas. Although the three subdivisions of the LP were not discriminated in the early studies, a recent study showed that the rat LPcm projected not only to the V2, but also to area TE2 and the dorsal perirhinal cortex (Shi \& Davis, 2001), which mostly corresponded to the postrhinal and temporal 
association areas in the present study.

Furthermore, single neuron labeling and PHA-L anterograde tracing experiments revealed that LP neurons reconstructed in the present study sent a small number of axon fibers to a variety of cortical areas, including areas 35 and 36, the retrosplenial, postsubicular, somatosensory, posterior parietal, auditory, motor, prelimbic and anterior cingulate areas (Table 1, 3). These results are also in good agreement with the previous reports on the widespread projections of LP neurons to the retrosplenial (Chandler et al., 1992; van Groen \& Wyss, 1992; Reep et al., 1994; Kamishina et al., 2009), perirhinal (Shi \& Davis, 2001), primary somatosensory (Chandler et al., 1992), posterior parietal (Chandler et al., 1992; Reep et al., 1994; Kamishina et al., 2009), medial agranular/Fr2 (secondary motor; Sukekawa, 1988; Hicks \& Huerta, 1991; Reep \& Corwin, 1999; Kamishina et al., 2009), and anterior cingulate areas (Kamishina et al., 2009). It is therefore presumed that visual information is transmitted simultaneously to many cortical areas by the broad variety of LP-cortical projections.

In contrast to the LPl and LPrm, LPcm neurons preferred the 'extravisual' areas to the visual ones. Since the LPcm receives intense inputs from the superficial layers of the superior colliculus (Perry, 1980; Mason \& Groos, 1981; Takahashi, 1985; Shi \& Davis, 2001; Masterson et al., 2009), the LPcm may be associated with visuospatial function. In the present study, all of the reconstructed LPcm neurons projected a large number of axon fibers 
to the postrhinal area (mean \pm S.D. $=58.0 \pm 17.5,32.3-75.1 \mathrm{~mm} /$ neuron; Table 1$)$, but not so many to the visual areas $(10.3 \pm 10.6,0-24.2 \mathrm{~mm} /$ neuron). The previous (Shi \& Davis, 2001) and present anterograde tracing experiments further showed that the main target area of the LPcm was the postrhinal area.

The postrhinal area is heavily interconnected with the V2 and V1 in the rodent brain (Mcdonald \& Mascagni, 1996; Agster \& Burwell, 2009; for review, see Furtak et al., 2007), and therefore, the area receives visual information from both the LPcm and visual areas. The postrhinal area also makes strong connection with the entorhinal area directly or indirectly through the perirhinal areas 35 and 36 (for review, see Furtak et al., 2007). These connections imply that the LPcm and postrhinal area may be involved in visuospatial processing, learning and memory. Actually, several lesion studies of the caudal temporal regions including the postrhinal area in the rat brain demonstrated the impairment in visual attention, visual discrimination and visually based learning (Meyer et al., 1986; Kolb et al., 1994; Wörtwein et al., 1994; Bucci et al., 2000, 2002; Bucci \& Burwell, 2004; Burwell et al., 2004). Thus, it seems reasonable that the LPcm as well as the postrhinal area take part in visuospatial function.

The present PHA-L anterograde tracing experiments showed that LPl and LPrm neurons sent axon fibers mainly to L2-5 and L1 in the visual areas, respectively. In the previous anterograde labeling studies, LP neurons projected their axon fibers to L1 and L3-4 
in the V2 (Hughes, 1977), or to L1 and L4 in the V2 and to L1 and L5 in the V1 (Herkenham, 1980), being mostly compatible with the present results. The present experiments of single neuron labeling further revealed that the axon varicosities of LPl neurons preferred the middle layers (L2-5) of the visual areas as their targets, whereas those of LPrm neurons favored L1 (Fig. 8, 9).

Core and matrix classification/organization of the thalamic nuclei has been originally proposed mainly in the sensory thalamus such as the ventral posterior nuclei, medial geniculate complex, and LGd (for review, see Jones, 1998, 2001). In the primates, core- and matrix-types neurons are chemically characterized by the expression of calcium-binding proteins. Core-type neurons are immunoreactive for PV and restricted in certain nuclei or subnuclei, whereas matrix-type neurons are positive for $\mathrm{CB}$ and distributed in all thalamic nuclei: core- and matrix-types neurons are intermingled in the dorsal thalamus. These two types of thalamic neurons also show the differences in the input-output connections. Core-type neurons receive subcortical inputs in a highly topographic fashion, and send axon fibers to the middle cortical layers (mainly L4) of the cerebral cortex. This input-output connection mostly displays one-to-one-correspondence, and has been assumed to convey the information accurately and precisely as driver inputs to the cortex. On the other hand, matrix-type neurons receive more diffuse subcortical inputs, and project wide-spreadly to the superficial layer (L1) of the cortex, suggesting that matrix-type neurons might be involved in 
more global cortical functions such as attention rather than focal activation.

The definition of core and matrix classification in the primates, however, cannot be directly applied to the rodents. In the rodent thalamic nuclei, no PV-immunoreactive neurons were found except the thalamic reticular nucleus (Rubio-Garrido et al., 2007, 2009; for review, see Jones, 1998; Clascá et al., 2012). Furthermore, both LPrm and LPl neurons were immunoreactive for $\mathrm{CB}$ in the present single neuron tracing study, although LPrm and LP1 neurons mainly projected to the L1 and middle cortical layers of the V2 and can be categorized into matrix- and core-types relay neurons, respectively. Hence, in the rodent thalamus, core- and matrix-types should be distinguished by cortical projections but not by chemical characteristics.

A similar issue was addressed in the rostral portion of the posterior thalamic nucleus (POm) of the rats in the previous study (Ohno et al., 2012). CB immunoreactivity was observed not only in posterior POm neurons projecting mainly to L1, but also in anterior POm neurons preferring the middle layers of the cerebral cortex. Accordingly, we divided them into core- and matrix-types neurons based on the target cortical layers. The previous and present results suggest that $\mathrm{CB}$ expression is not a helpful marker, but only laminar preference is useful in determining the core-matrix organization in some thalamic nuclei of the rat brain (Rubio-Garrido et al., 2007, 2009; for review, see Clascá et al., 2012). We therefore concluded that LPl and LPrm neurons are classified into core- and matrix-types 
thalamic neurons on the basis of thalamocortical innervation. In addition, it should be noticed that the axonal arborizations of matrix-type neurons (LPrm and posterior POm) display a widespread distribution even at a single neuron level, as compared with those of core-type neurons (LPl and anterior POm).

The LP1 is known to receive cortical inputs not only from the V2 but also from the V1 (Mason \& Groos, 1981; Takahashi, 1985; Bourassa \& Deschênes, 1995), and then sent a large number of axon fibers to the middle layers of the V2 (Fig. 8). Thus, the LPl is considered to be one of the higher-order relay thalamic nuclei, which have been proposed by Sherman and Guillery (for review, see Sherman \& Guillery, 2006; Sherman, 2007, 2012; Varela, 2014). The anterior POm is also classified as the higher-order thalamic nuclei, since the anterior POm receives information from the primary somatosensory area, and transmits it to the higher-order sensory areas (for review, see Sherman \& Guillery, 2006; Varela, 2014). In addition, 5 of $7 \mathrm{LPl}$ neurons examined in the present study formed at least two axonal clusters in the V2, whereas 3 of 5 anterior POm neurons formed multiple axonal clusters in the sensory areas (Ohno et al., 2012). The previous and present findings suggest that the LPl share similar characteristics with the anterior POm as the higher-order thalamic nuclei.

It has been reported that the rat V2 contains 5-10 retinotopic representations of visual space (Montero et al., 1973; Espinoza \& Thomas, 1983; Montero, 1993). Furthermore, multiple V1-to-V2 projections have been confirmed in the rat and mouse visual 
areas (Coogan \& Burkhalter, 1993; Wang \& Burkhalter, 2007). More recently, imaging techniques with calcium and flavoprotein fluorescence have clearly shown the presence of discrete subregions in the mouse V2 (Andermann et al., 2011; Marshel et al., 2011; Tohmi et al., 2014). These previous reports support the idea that the rodent V2 is composed of multiple functionally distinct subregions. Taking account of the multiple innervation of the V2 by the LP1 as described above, LP1 neurons may control the functionally different subregions of the V2 simultaneously by transmitting the information converging mainly from the visual areas. 


\section{Acknowledgements}

This work was supported by Grant-in-Aid for Scientific Research on Innovative Areas "Neural Diversity and Neocortical Organization" (25123709 to H.H.), "Foundation of Synapse and Neurocircuit Pathology" (22110007 to H.H.) and "Mesoscopic Neurocircuitry" (23115101 to T.K.), and Grants-in-Aid for Scientific Research (24500408 to H.H.; 24500408 to T.F.; 25250006 to T.K.) from The Ministry of Education, Culture, Sports, Science and Technology (MEXT) and Japan Society for the Promotion of Science (JSPS).

\section{Conflict of Interest}

The authors declare no competing financial interests. 


\section{Abbreviations}

$\mathrm{A} 1$, primary auditory area; $\mathrm{AC}$, anterior cingulate area; $\mathrm{CB}$, calbindin $\mathrm{D}-28 \mathrm{k} ; \mathrm{CPu}$, caudate-putamen; LGd, dorsal lateral geniculate nucleus; LP, lateral posterior thalamic nucleus; LPcm, caudomedial portion of the LP; LPl, lateral portion of the LP; LPrm, rostromedial portion of the LP; M2, secondary motor area; POm, rostral sector of the posterior nucleus; POR, postrhinal area; PoS, postsubiculum; PP, posterior parietal area; RS, retrosplenial area; Rt, thalamic reticular nucleus; TEa, temporal association area; V1, primary visual area; V2, secondary visual area; V21, lateral region of the V2; V2m, medial region of the V2; VPM, ventral posteromedial nucleus. 


\section{References}

Agster, K.L. \& Burwell, R.D. (2009) Cortical efferents of the perirhinal, postrhinal, and entorhinal cortices of the rat. Hippocampus, 19, 1159-1186.

Ahmed, A.K., Dong, K. \& Yamadori, T. (1995) A retrograde double-labelling study of retinal ganglion cells that project ipsilaterally to vLGN and LPN rather than dLGN and SC, in albino rat. Brain Res., 674, 275-282.

Andermann, M.L., Kerlin, A.M., Roumis, D.K., Glickfeld, L.L. \& Reid, R.C. (2011) Functional specialization of mouse higher visual cortical areas. Neuron, 72, 1025-1039.

Bourassa, J. \& Deschênes, M. (1995) Corticothalamic projections from the primary visual cortex in rats: a single fiber study using biocytin as an anterograde tracer. Neuroscience, 66, 253-263.

Bucci, D.J. \& Burwell, R.D. (2004) Deficits in attentional orienting following damage to the perirhinal or postrhinal cortices. Behav. Neurosci., 118, 1117-1122.

Bucci, D.J., Phillips, R.G. \& Burwell, R.D. (2000) Contributions of postrhinal and perirhinal cortex to contextual information processing. Behav. Neurosci., 114, 882-894.

Bucci, D.J., Saddoris, M.P. \& Burwell, R.D. (2002) Contextual fear discrimination is impaired by damage to the postrhinal or perirhinal cortex. Behav. Neurosci., 116, 479-488.

Burwell, R.D., Bucci, D.J., Sanborn, M.R. \& Jutras, M.J. (2004) Perirhinal and postrhinal 
contributions to remote memory for context. J. Neurosci., 24, 11023-11028.

Chandler, H.C., King, V., Corwin, J.V. \& Reep, R.L. (1992) Thalamocortical connections of rat posterior parietal cortex. Neurosci. Lett., 143, 237-242.

Clascá F, Rubio-Garrido P, Jabaudon D. (2012) Unveiling the diversity of thalamocortical neuron subtypes. Eur J Neurosci., 35, 1524-1532.

Coogan, T.A. \& Burkhalter, A. (1993) Hierarchical organization of areas in rat visual cortex. J. Neurosci., 13, 3749-3772.

Diamond, I.T. (1976) Organization of the visual cortex: comparative anatomical and behavioral studies. Fed. Proc., 35, 60-67.

Diamond, I.T. \& Hall, W.C. (1969) Evolution of neocortex. Science, 164, 251-262.

Espinoza, S.G. \& Thomas, H.C. (1983) Retinotopic organization of striate and extrastriate visual cortex in the hooded rat. Brain Res., 272, 137-144.

Fujiyama, F., Sohn, J., Nakano, T., Furuta, T., Nakamura, K.C., Matsuda, W. \& Kaneko, T. (2011) Exclusive and common targets of neostriatofugal projections of rat striosome neurons: a single neuron-tracing study using a viral vector. Eur. J. Neurosci., 33, $668-677$.

Funaki, S., Meguro, R., Abe, H. \& Norita, M. (1998) The organization of the thalamostriatal projection from the lateral posterior thalamic nuclear complex (LP) in the pigmented rat. Neurobiology (Bp.), 6, 273-294. 
Furtak, S.C., Wei, S.M., Agster, K.L. \& Burwell, R.D. (2007) Functional neuroanatomy of the parahippocampal region in the rat: the perirhinal and postrhinal cortices. Hippocampus, 17, 709-722.

Furuta, T., Kaneko, T. \& Deschênes, M. (2009) Septal neurons in barrel cortex derive their receptive field input from the lemniscal pathway. J. Neurosci., 29, 4089-4095.

Furuta, T., Tomioka, R., Taki, K., Nakamura, K., Tamamaki, N. \& Kaneko, T. (2001) In vivo transduction of central neurons using recombinant Sindbis virus: Golgi-like labeling of dendrites and axons with membrane-targeted fluorescent proteins. J. Histochem. Cytochem., 49, 1497-1508.

Goodman, D.C., Bogdasarian, R.S. \& Horel, J.A. (1973) Axonal sprouting of ipsilateral optic tract following opposite eye removal. Brain Behav. Evol., 8, 27-50.

Herkenham, M. (1980) Laminar organization of thalamic projections to the rat neocortex. Science, 207, 532-535.

Hicks, R.R. \& Huerta, M.F. (1991) Differential thalamic connectivity of rostral and caudal parts of cortical area Fr2 in rats. Brain Res., 568, 325-329.

Hioki, H., Fujiyama, F., Taki, K., Tomioka, R., Furuta, T., Tamamaki, N. \& Kaneko, T. (2003) Differential distribution of vesicular glutamate transporters in the rat cerebellar cortex. Neuroscience, 117, 1-6.

Hioki, H., Nakamura, H., Ma, Y.F., Konno, M., Hayakawa, T., Nakamura, K.C., Fujiyama, F. 
\& Kaneko, T. (2010) Vesicular glutamate transporter 3-expressing nonserotonergic projection neurons constitute a subregion in the rat midbrain raphe nuclei. J. Comp. Neurol., 518, 668-686.

Hioki, H., Okamoto, S., Konno, M., Kameda, H., Sohn, J., Kuramoto, E., Fujiyama, F. \& Kaneko, T. (2013) Cell type-specific inhibitory inputs to dendritic and somatic compartments of parvalbumin-expressing neocortical interneuron. J. Neurosci., 33, $544-555$.

Hughes, H.C. (1977) Anatomical and neurobehavioral investigations concerning the thalamo-cortical organization of the rat's visual system. J. Comp. Neurol., 175, 311-336. Jones, E.G. (1998) Viewpoint: the core and matrix of thalamic organization. Neuroscience, 85, $331-345$.

Jones, E.G. (2001) The thalamic matrix and thalamocortical synchrony. Trends Neurosci., 24, $595-601$.

Jones, E.G. (2007) The Thalamus. Cambridge University Press, Cambridge (UK).

Kamishina, H., Conte, W.L., Patel, S.S., Tai, R.J., Corwin, J.V. \& Reep, R.L. (2009) Cortical connections of the rat lateral posterior thalamic nucleus. Brain Res., 1264, 39-56.

Kamishina, H., Yurcisin, G.H., Corwin, J.V. \& Reep, R.L. (2008) Striatal projections from the rat lateral posterior thalamic nucleus. Brain Res., 1204, 24-39.

Kolb, B., Buhrmann, K., McDonald, R. \& Sutherland, R.J. (1994) Dissociation of the medial 
prefrontal, posterior parietal, and posterior temporal cortex for spatial navigation and recognition memory in the rat. Cereb. Cortex, 4, 664-680.

Koshimizu, Y., Fujiyama, F., Nakamura, K.C., Furuta, T. \& Kaneko, T. (2013) Quantitative analysis of axon bouton distribution of subthalamic nucleus neurons in the rat by single neuron visualization with a viral vector. J. Comp. Neurol., 521, 2125-2146.

Kuramoto, E., Furuta, T., Nakamura, K.C., Unzai, T., Hioki, H. \& Kaneko, T. (2009) Two types of thalamocortical projections from the motor thalamic nuclei of the rat: a single neuron-tracing study using viral vectors. Cereb. Cortex, 19, 2065-2077.

Kuramoto, E., Ohno, S., Furuta, T., Unzai, T., Tanaka, Y.R., Hioki, H. \& Kaneko, T. (2015) Ventral medial nucleus neurons send thalamocortical afferents more widely and more preferentially to layer 1 than neurons of the ventral anterior-ventral lateral nuclear complex in the rat. Cereb. Cortex, 25, 221-235.

Marshel, J.H., Garrett, M.E., Nauhaus, I. \& Callaway, E.M. (2011) Functional specialization of seven mouse visual cortical areas. Neuron, 72, 1040-1054.

Mason, R. \& Groos, G.A. (1981) Cortico-recipient and tecto-recipient visual zones in the rat's lateral posterior (pulvinar) nucleus: an anatomical study. Neurosci. Lett., 25, 107-112.

Masterson, S.P., Li, J. \& Bickford, M.E. (2009) Synaptic organization of the tectorecipient zone of the rat lateral posterior nucleus. J. Comp. Neurol., 515, 647-663.

Matsuda, W., Furuta, T., Nakamura, K.C., Hioki, H., Fujiyama, F., Arai, R. \& Kaneko, T. 
(2009) Single nigrostriatal dopaminergic neurons form widely spread and highly dense axonal arborizations in the neostriatum. J. Neurosci., 29, 444-453.

Mcdonald, A.J. \& Mascagni, F. (1996) Cortico-cortical and cortico-amygdaloid projections of the rat occipital cortex: a Phaseolus vulgaris leucoagglutinin study. Neuroscience, 71, $37-54$

Meyer, P.M., Meyer, D.R. \& Cloud, M.D. (1986) Temporal neocortical injuries in rats impair attending but not complex visual processing. Behav. Neurosci., 100, 845-851.

Montero, V.M. (1993) Retinotopy of cortical connections between the striate cortex and extrastriate visual areas in the rat. Exp. Brain Res., 94, 1-15.

Montero, V.M., Rojas, A. \& Torrealba, F. (1973) Retinotopic organization of striate and peristriate visual cortex in the albino rat. Brain Res., 53, 197-201.

Moriyoshi K, Richards LJ, Akazawa C, O'Leary DD, Nakanishi S. (1996) Labeling neural cells using adenoviral gene transfer of membrane-targeted GFP. Neuron, 16, 255-260.

Nishino, E., Yamada, R., Kuba, H., Hioki, H., Furuta, T., Kaneko, T. \& Ohmori, H. (2008) Sound-intensity-dependent compensation for the small interaural time difference cue for sound source localization. J. Neurosci., 28, 7153-7164.

Noseda, R., Jakubowski, M., Kainz, V., Borsook, D. \& Burstein, R. (2011) Cortical projections of functionally identified thalamic trigeminovascular neurons: implications for migraine headache and its associated symptoms. J. Neurosci., 31, 14204-14217. 
Noseda, R., Kainz, V., Jakubowski, M., Gooley, J.J., Saper, C.B., Digre, K. \& Burstein, R. (2010) A neural mechanism for exacerbation of headache by light. Nat. Neurosci., 13, $239-245$.

Ohno, S., Kuramoto, E., Furuta, T., Hioki, H., Tanaka, Y.R., Fujiyama, F., Sonomura, T., Uemura, M., Sugiyama, K. \& Kaneko, T. (2012) A morphological analysis of thalamocortical axon fibers of rat posterior thalamic nuclei: a single neuron tracing study with viral vectors. Cereb. Cortex, 22, 2840-2857.

Olavarria, J. (1979) A horseradish peroxidase study of the projections from the latero-posterior nucleus to three lateral peristriate areas in the rat. Brain Res., 173, $137-141$.

Parnavelas, J.G., Chatzissavidou, A. \& Burne, R.A. (1981) Subcortical projections to layer I of the visual cortex, area 17, of the rat. Exp. Brain Res., 41, 184-187.

Paxinos, G. \& Watson, C. (2007) The Rat Brain in Stereotaxic Coordinates. Academic Press, San Diego.

Perry, V.H. (1980) A tectocortical visual pathway in the rat. Neuroscience, 5, 915-927.

Reep, R.L., Chandler, H.C., King, V. \& Corwin, J.V. (1994) Rat posterior parietal cortex: topography of corticocortical and thalamic connections. Exp. Brain Res., 100, 67-84.

Reep, R.L. \& Corwin, J.V. (1999) Topographic organization of the striatal and thalamic connections of rat medial agranular cortex. Brain Res., 841, 43-52. 
Rubio-Garrido P, Pérez-de-Manzo F, Clascá F. (2007) Calcium-binding proteins as markers of layer-I projecting vs. deep layer-projecting thalamocortical neurons: a double-labeling analysis in the rat. Neuroscience, 149, 242-250.

Rubio-Garrido, P., Pérez-de-Manzo, F., Porrero, C., Galazo, M.J. \& Clascá, F. (2009) Thalamic input to distal apical dendrites in neocortical layer 1 is massive and highly convergent. Cereb. Cortex, 19, 2380-2395.

Sanderson, K.J., Dreher, B. \& Gayer, N. (1991) Prosencephalic connections of striate and extrastriate areas of rat visual cortex. Exp. Brain Res., 85, 324-334.

Schneider, G.E. (1969) Two visual systems. Science, 163, 895-902.

Schobber, W. (1981) [Efferent and afferent connections of the nucleus lateralis posterior thalami ("pulvinar") in the albino rats (author's transl)]. Z. Mikrosk. Anat. Forsch., 95, $827-844$.

Serizawa, M., McHaffie, J.G., Hoshino, K. \& Norita, M. (1994) Corticostriatal and corticotectal projections from visual cortical areas 17,18 and 18a in the pigmented rat. Arch. Histol. Cytol., 57, 493-507.

Sherman SM. (2007) The thalamus is more than just a relay. Curr Opin Neurobiol., 17, 417-422.

Sherman SM. (2012) Thalamocortical interactions. Curr Opin Neurobiol., 22, 575-579.

Sherman, S.M. \& Guillery, R.W. (2006) Exploring the Thalamus and its Role in Cortical 
Function. MIT Press, Cambridge (MA).

Shi, C. \& Davis, M. (2001) Visual pathways involved in fear conditioning measured with fear-potentiated startle: behavioral and anatomic studies. J. Neurosci., 21, 9844-9855.

Sholl, D.A. (1953) Dendritic organization in the neurons of the visual and motor cortices of the cat. J. Anat., 87, 387-406.

Sukekawa, K. (1988) Reciprocal connections between medial prefrontal cortex and lateral posterior nucleus in rats. Brain Behav. Evol., 32, 246-251.

Swanson, L.W. (2004) Brain Maps: Structure of the Rat Brain. Elsevier Academic Press, Amsterdam.

Takahashi, T. (1985) The organization of the lateral thalamus of the hooded rat. J. Comp. Neurol., 231, 281-309.

Tamamaki, N., Nakamura, K., Furuta, T., Asamoto, K. \& Kaneko, T. (2000) Neurons in Golgi-stain-like images revealed by GFP-adenovirus infection in vivo. Neurosci. Res., 38 , $231-236$.

Tanaka YR, Tanaka YH, Konno M, Fujiyama F, Sonomura T, Okamoto-Furuta K, Kameda H, Hioki H, Furuta T, Nakamura KC, Kaneko T. (2011) Local connections of excitatory neurons to corticothalamic neurons in the rat barrel cortex. $J$ Neurosci., 31, 1822318236.

Tohmi, M., Meguro, R., Tsukano, H., Hishida, R. \& Shibuki, K. (2014) The extrageniculate 
visual pathway generates distinct response properties in the higher visual areas of mice.

Curr. Biol., 24, 587-597.

Varela C. (2014) Thalamic neuromodulation and its implications for executive networks.

Front Neural Circuits., 8, 69.

van Groen, T. \& Wyss, J.M. (1992) Connections of the retrosplenial dysgranular cortex in the rat. J. Comp. Neurol., 315, 200-216.

Wang, Q. \& Burkhalter, A. (2007) Area map of mouse visual cortex. J. Comp. Neurol., 502, $339-357$.

Wörtwein, G., Mogensen, J., Williams, G., Carlos, J.H. \& Divac, I. (1994) Cortical area in the rat that mediates visual pattern discrimination. Acta. Neurobiol. Exp. (Wars.), 54, $365-376$.

Yamadori, T. (1977) An experimental anatomical study on the optic nerve fibers in the rat by using a new selective silver impregnation technique: termination of the main optic tract. Okajimas Folia Anat. Jpn., 54, 229-245.

Zilles, K. (1985) The Cortex of the Rat. A Stereotaxic Atlas. Springer-Verlag, Berlin. 
Table 1. The length [mm] of varicose axons from LP neurons in each cortical area and striatum.

\begin{tabular}{|c|c|c|c|c|c|c|c|c|c|c|c|c|c|c|c|c|}
\hline \multirow{2}{*}{$\begin{array}{l}\text { Cortical } \\
\text { areas }\end{array}$} & \multicolumn{7}{|c|}{ LPl neurons } & \multicolumn{4}{|c|}{ LPrm neurons } & \multicolumn{5}{|c|}{ LPcm neurons } \\
\hline & 1 & 2 & 3 & 4 & 5 & 6 & 7 & 8 & 9 & 10 & 11 & 12 & 13 & 14 & 15 & 16 \\
\hline V1 & 0 & 0 & 39.9 & 15.8 & 0 & $\underline{81.1}$ & 47.2 & $\underline{68.9}$ & 3.9 & 2.4 & $\underline{51.1}$ & 0 & 0.4 & 6.7 & 4.5 & 0 \\
\hline $\mathrm{V} 2$ & $\underline{169.2}$ & $\underline{55.6}$ & $\underline{60.1}$ & $\underline{120.7}$ & $\underline{114.4}$ & 19.5 & $\underline{82.4}$ & $\underline{94.7}$ & 42.7 & 46.8 & 48.6 & 0 & 16.6 & 17.5 & 5.9 & 0 \\
\hline $\mathrm{RS}$ & 0 & 38.4 & 0 & 33.5 & 0 & 0 & 0 & 5.9 & 0 & 0 & 3.7 & 0 & 0 & 0 & 0 & 0 \\
\hline $\mathrm{TEa}$ & 0 & 0 & 0 & 1.1 & 0 & 0 & 0 & 0 & $\underline{55.2}$ & 6.6 & 0 & 3.6 & 42.7 & 9.0 & 36.4 & 2.0 \\
\hline POR & 0 & 0 & 7.0 & 0 & 0 & 0 & 0 & 5.1 & 18.1 & 47.9 & 0 & 49.1 & 29.6 & $\underline{71.3}$ & $\underline{62.3}$ & $\underline{75.1}$ \\
\hline 36 & 0 & 0 & 0 & 0 & 0 & 0 & 0 & 0 & 0 & 0 & 0 & 49.5 & 0 & 0 & 0 & 0 \\
\hline 35 & 0 & 0 & 0 & 0 & 0 & 0 & 0 & 0 & 0 & 0 & 0 & 5.2 & 0 & 0 & 0 & 0 \\
\hline PoS & 0 & 2.1 & 0 & 8.1 & 0 & 0 & 0.7 & 0 & 0 & 0 & 0 & 0 & 0 & 0 & 0 & 0 \\
\hline $\mathrm{S} 1 / \mathrm{S} 2$ & 5.1 & 0 & 0 & 0 & 0 & 5.1 & 7.3 & 0.6 & 0 & 0 & 0 & 0 & 0 & 0 & 0 & 0 \\
\hline PP & 0 & 0 & 0 & 0 & 0 & 0 & 0 & 4.4 & 0 & 0 & 6.3 & 0 & 0 & 0 & 0 & 0 \\
\hline $\mathrm{A} 1 / \mathrm{A} 2$ & 0 & 0 & 0 & 0 & 0 & 0.5 & 0 & 0 & 1.6 & 0 & 5.6 & 0 & 0 & 0 & 0 & 0 \\
\hline M1 & 0 & 0 & 0 & 0 & 0 & 0 & 0 & 0 & 0 & 0 & 5.3 & 0 & 0 & 0 & 0 & 0 \\
\hline M2 & 0 & 0 & 0 & 0 & 0 & 0 & 0 & 3.9 & 0 & 0 & 44.0 & 0 & 0 & 0 & 0 & 0 \\
\hline PL & 0 & 0 & 0 & 0 & 0 & 0 & 0 & 2.9 & 0 & 0 & 0 & 0 & 0 & 0 & 0 & 0 \\
\hline $\mathrm{AC}$ & 0 & 0 & 0 & 0 & 0 & 0 & 0 & 16.5 & 0 & 0 & 30.4 & 0 & 0 & 0 & 0 & 0 \\
\hline $\mathrm{CPu}$ & 34.0 & 8.3 & 0 & 0 & 12.2 & 0 & 4.8 & 8.5 & 26.4 & 0 & 5.0 & 34.0 & 3.8 & 0 & 6.7 & 28.8 \\
\hline
\end{tabular}

Underlines and boldfaces indicate the target areas containing axon fibers of more than 50 and $25 \mathrm{~mm}$, respectively. 35, area 35; 36, area 36; A2, secondary auditory area; M1, primary motor area; PL, prelimbic area; S1, primary somatosensory area; S2, secondary somatosensory area. 
Table 2. The length [mm] of varicose axons from LP neurons in each layer of the cerebral cortex.

\begin{tabular}{|c|c|c|c|c|c|}
\hline \multirow{2}{*}{ Neuron } & \multicolumn{5}{|c|}{ Length of varicose axons } \\
\hline & Layer 1 & Layers 2-4 & Layer 5 & Layer 6 & Total \\
\hline \multicolumn{6}{|l|}{ LPl } \\
\hline 1 & $12.1(7.0 \%)$ & $93.8(53.9 \%)$ & $62.7(36.0 \%)$ & $5.4(3.1 \%)$ & 174.1 \\
\hline 2 & $18.0(18.7 \%)$ & $50.5(52.6 \%)$ & $25.5(26.5 \%)$ & $2.1(2.1 \%)$ & 96.1 \\
\hline 3 & $17.2(16.1 \%)$ & $59.6(56.1 \%)$ & $28.8(27.1 \%)$ & $0.8(0.7 \%)$ & 106.4 \\
\hline 4 & $48.8(27.2 \%)$ & $80.2(44.8 \%)$ & $49.4(27.5 \%)$ & $0.9(0.5 \%)$ & 179.2 \\
\hline 5 & $9.6(8.4 \%)$ & $70.3(61.5 \%)$ & $34.1(29.9 \%)$ & $0.3(0.3 \%)$ & 114.4 \\
\hline 6 & $9.8(9.2 \%)$ & $49.1(46.2 \%)$ & $43.0(40.5 \%)$ & $4.3(4.0 \%)$ & 106.2 \\
\hline 7 & $28.3(20.6 \%)$ & $19.2(14.0 \%)$ & $88.5(64.4 \%)$ & $1.5(1.1 \%)$ & 137.5 \\
\hline \multicolumn{6}{|l|}{ LPrm } \\
\hline 8 & $158.1(77.9 \%)$ & $20.2(9.9 \%)$ & $21.4(10.6 \%)$ & $3.2(1.6 \%)$ & 202.9 \\
\hline 9 & $68.7(56.6 \%)$ & $27.6(22.7 \%)$ & $23.6(19.4 \%)$ & $1.6(1.3 \%)$ & 121.4 \\
\hline 10 & $28.0(27.0 \%)$ & $23.1(22.2 \%)$ & $50.2(48.4 \%)$ & $2.5(2.4 \%)$ & 103.8 \\
\hline 11 & $65.3(33.5 \%)$ & $69.1(35.4 \%)$ & $50.0(25.6 \%)$ & $10.7(5.5 \%)$ & 195.1 \\
\hline \multicolumn{6}{|l|}{ LPcm } \\
\hline 12 & $10.7(10.0 \%)$ & $48.7(45.3 \%)$ & $48.0(44.7 \%)$ & $0(0 \%)$ & 107.4 \\
\hline 13 & $43.0(48.2 \%)$ & $22.6(25.3 \%)$ & $22.2(24.8 \%)$ & $1.5(1.7 \%)$ & 89.3 \\
\hline 14 & $7.4(7.1 \%)$ & $55.7(53.3 \%)$ & $38.7(37.0 \%)$ & $2.7(2.6 \%)$ & 104.5 \\
\hline 15 & $30.2(27.7 \%)$ & $42.1(38.6 \%)$ & $35.1(32.2 \%)$ & $1.6(1.5 \%)$ & 109.1 \\
\hline 16 & $29.1(37.8 \%)$ & $22.9(29.7 \%)$ & $21.5(27.8 \%)$ & $3.6(4.7 \%)$ & 77.1 \\
\hline
\end{tabular}


Table 3. The semiquantitative evaluation of anterogradely labeled axon fibers after injections of

\section{PHA-L into the LP.}

\begin{tabular}{|c|c|c|c|c|c|c|c|c|c|c|c|c|c|c|c|c|c|c|c|c|c|c|c|c|}
\hline \multirow{2}{*}{ Case } & \multirow{2}{*}{$\begin{array}{l}\text { Injection } \\
\text { site }\end{array}$} & \multicolumn{23}{|c|}{ Target areas } \\
\hline & & V1 & V2 & RS & TEa & POR & 36 & 35 & PoS & $\mathrm{S} 1 / 2$ & PP & $\mathrm{A} 1 / 2$ & M1 & M2 & PL & $\mathrm{AC}$ & Ent & $\mathrm{PaS}$ & $\operatorname{PrS}$ & Pir & Orb & IL & FrA & Ins \\
\hline PH1 & LPl & ++ & +++ & + & + & + & - & - & - & ++ & ++ & + & - & + & - & + & + & - & - & - & + & + & - & - \\
\hline $\mathrm{PH} 2$ & LPl & ++ & +++ & ++ & ++ & ++ & ++ & + & - & + & ++ & + & + & ++ & ++ & ++ & ++ & + & - & - & ++ & + & - & - \\
\hline PH3 & LPl & ++ & +++ & - & + & ++ & + & - & - & + & ++ & + & - & - & - & - & - & - & - & - & - & - & - & - \\
\hline PH4 & LPl & ++ & +++ & ++ & ++ & ++ & + & + & ++ & + & ++ & ++ & - & - & - & - & + & - & - & - & - & - & - & - \\
\hline PH5 & $\mathrm{LPl}$ & ++ & +++ & ++ & ++ & ++ & + & + & + & + & ++ & ++ & - & + & - & - & ++ & - & - & - & ++ & - & - & - \\
\hline PH6 & LPl & ++ & +++ & + & ++ & ++ & + & - & - & - & ++ & - & - & - & - & ++ & + & - & - & - & + & - & - & - \\
\hline PH7 & LPl-LPrm & ++ & +++ & ++ & ++ & ++ & + & + & + & ++ & ++ & + & + & + & + & ++ & + & - & - & - & + & + & - & - \\
\hline PH8 & LPl-LPrm & ++ & +++ & ++ & ++ & ++ & + & + & ++ & - & - & ++ & + & + & - & + & - & - & + & - & - & - & - & - \\
\hline PH9 & LPl-LPrm & ++ & +++ & + & ++ & ++ & ++ & ++ & + & - & ++ & ++ & + & ++ & ++ & ++ & ++ & - & - & - & ++ & - & - & - \\
\hline PH10 & LPl-LPrm & + & +++ & + & ++ & +++ & ++ & ++ & - & - & + & + & - & - & - & - & + & - & - & - & - & - & - & - \\
\hline PH11 & LPrm & ++ & +++ & ++ & +++ & +++ & ++ & + & +++ & ++ & ++ & + & + & ++ & ++ & +++ & ++ & ++ & - & - & ++ & + & - & - \\
\hline PH12 & LPrm & ++ & +++ & ++ & ++ & ++ & ++ & + & ++ & +++ & +++ & ++ & ++ & +++ & ++ & +++ & + & ++ & + & - & - & - & ++ & + \\
\hline PH13 & LPrm & + & +++ & ++ & ++ & ++ & ++ & ++ & - & + & ++ & ++ & + & ++ & ++ & ++ & + & ++ & - & - & ++ & - & - & - \\
\hline PH14 & LPrm & ++ & +++ & ++ & +++ & +++ & ++ & ++ & ++ & + & ++ & ++ & + & + & - & ++ & ++ & - & - & - & - & - & - & - \\
\hline PH15 & LPrm & + & +++ & ++ & ++ & + & + & - & - & - & ++ & + & + & + & + & + & - & - & - & - & + & - & - & - \\
\hline PH16 & $\mathrm{LPcm}$ & + & ++ & ++ & +++ & +++ & ++ & ++ & - & - & + & ++ & - & - & - & - & ++ & - & - & - & - & - & - & - \\
\hline PH17 & $\mathrm{LPcm}$ & + & +++ & + & +++ & +++ & ++ & + & - & + & + & + & + & + & - & - & ++ & - & - & + & + & - & - & + \\
\hline PH18 & $\mathrm{LPcm}$ & + & +++ & + & +++ & ++ & + & - & + & - & + & ++ & - & - & - & - & - & - & - & - & - & - & - & - \\
\hline PH19 & $\mathrm{LPcm}$ & + & ++ & - & +++ & +++ & ++ & + & - & - & + & + & - & - & - & - & + & - & - & - & - & - & - & - \\
\hline PH20 & $\mathrm{LPcm}$ & + & +++ & + & +++ & +++ & ++ & ++ & - & - & - & + & - & - & - & - & + & - & - & - & - & - & - & - \\
\hline PH21 & $\mathrm{LPcm}$ & + & ++ & + & ++ & ++ & ++ & + & - & + & + & ++ & - & - & ++ & + & + & - & - & - & - & - & - & - \\
\hline
\end{tabular}

The evaluation was performed with internal standards: +++, V2 of PH1; ++, V1 of PH1; +, POR of PH1 (see Figure 7A for more detail). 35, area 35; 36, area 36; A2, secondary auditory area; Ent, entorhinal area; FrA, frontal association area; IL, infralimbic area; Ins, insular area; M1, primary motor area; Orb, orbital area; PaS, parasubiculum; Pir, piriform area; PL, prelimbic area; PrS, presubiculum; S1, primary somatosensory area; $\mathrm{S} 2$, secondary somatosensory area. 

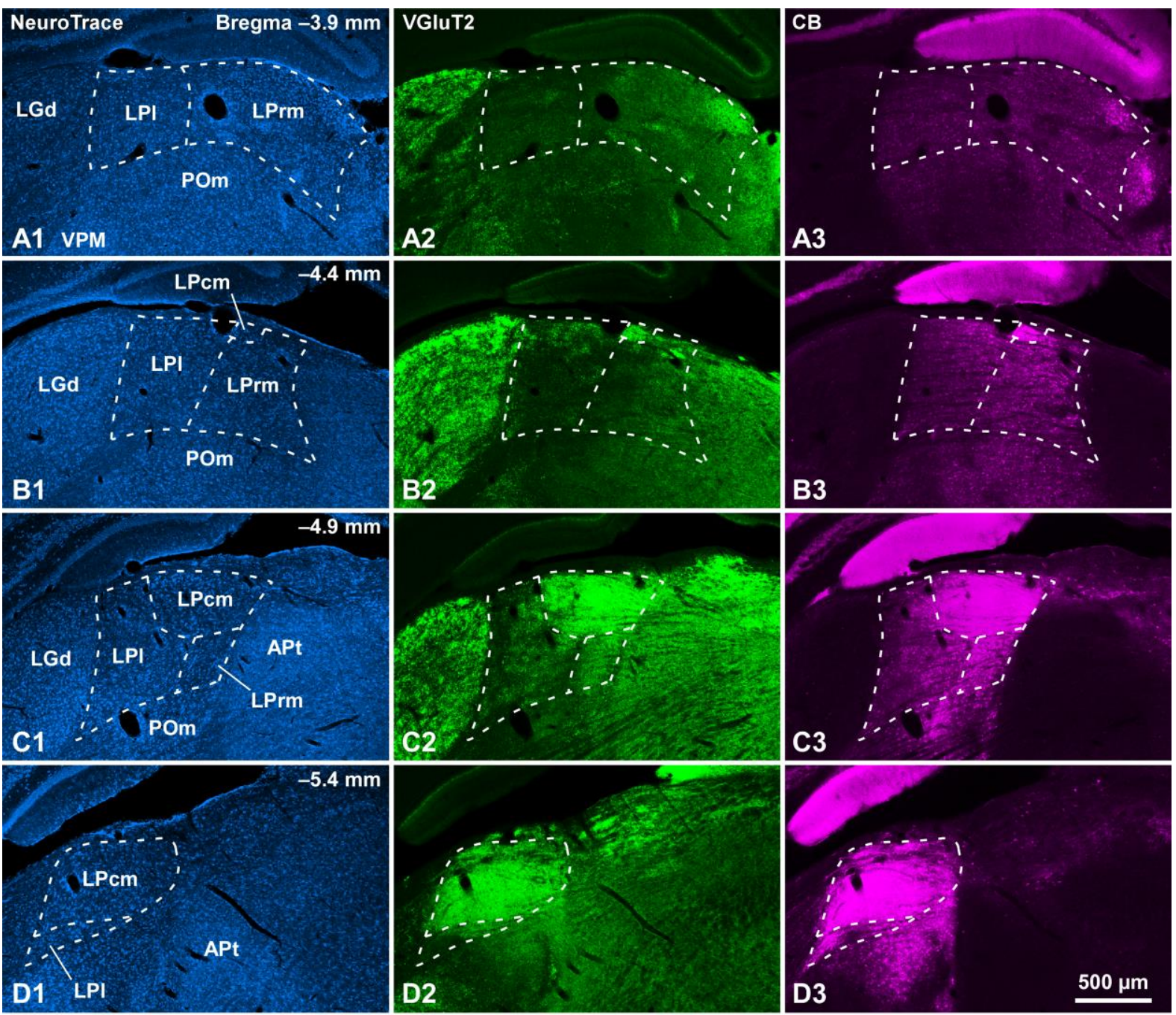

Figure 1

Figure 1. Cytoarchitecture and immunoreactivities for CB and VGluT2 in the rat LP. By reference to Nissl-like staining with NeuroTrace red (pseudo-colored blue; A1, B1, C1, D1), the LP was divided into three subregions, LPl, LPrm and LPcm in the coronal plane (left, lateral; right, medial) arranged from the rostral (A1) to caudal portion (D1). VGluT2 and CB were visualized by AF488 (green; A2, B2, C2, D2) and AF647 (magenta; A3, B3, C3, D3), respectively. Those immunoreactivities were more intense in the LPcm than in the LP1 and LPrm, and mostly overlapped in the LPcm (A2,3, B2,3, C2,3, D2,3). APt, anterior 
pretectal nucleus; POm, rostral sector of the posterior nucleus; VPM, ventral posteromedial nucleus. Scale bar in (D3) applies to all the figures. 


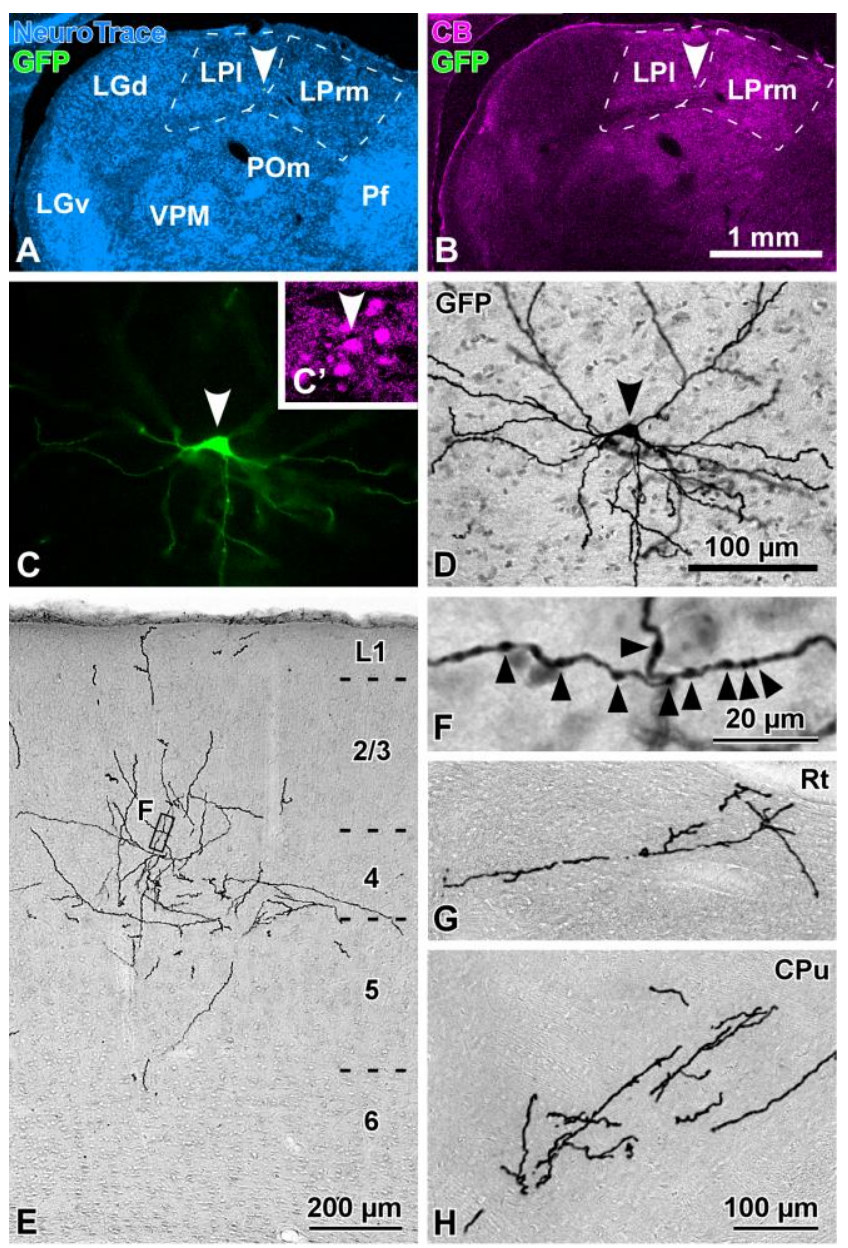

Figure 2

Figure 2. Visualization of single LP neurons with Sindbis virus vectors. When a neuron infected with palGFP (arrowheads in A-C) or pal-mRFP Sindbis virus was found in the LP under a fluorescence microscope, the section containing the labeled neuron was counterstained with NeuroTrace red (blue; A) or NeuroTrace green, respectively, and immunolabeled for CB (AF647; magenta; B, C'). After the location of the infected neuron was identified, the labeled neuron was visualized by immunoperoxidase staining with anti-GFP (D-H) or anti-mRFP antibody. The axons of single-labeled LP neurons were observed in the V2 (E, F), thalamic reticular nucleus (Rt; G), and caudate-putamen $(\mathrm{CPu} ; \mathrm{H})$. 
Arrowheads in $\mathrm{F}$ indicate the axon varicosities which were located at a focal plane of the microscope. To determine cortical areas and layers, we counterstained sections for Nissl with Cresyl violet after the reconstruction of the axonal arborizations. The counterstaining was not clear in the figure since the Cresyl violet color was photographically suppressed with a 420-nm-centered band path filter. LGv, ventral lateral geniculate nucleus; Pf, parafascicular nucleus. Scale bar in B applies to A, B; that in D to C, C', D; that in $\mathrm{H}$ to G, H. 

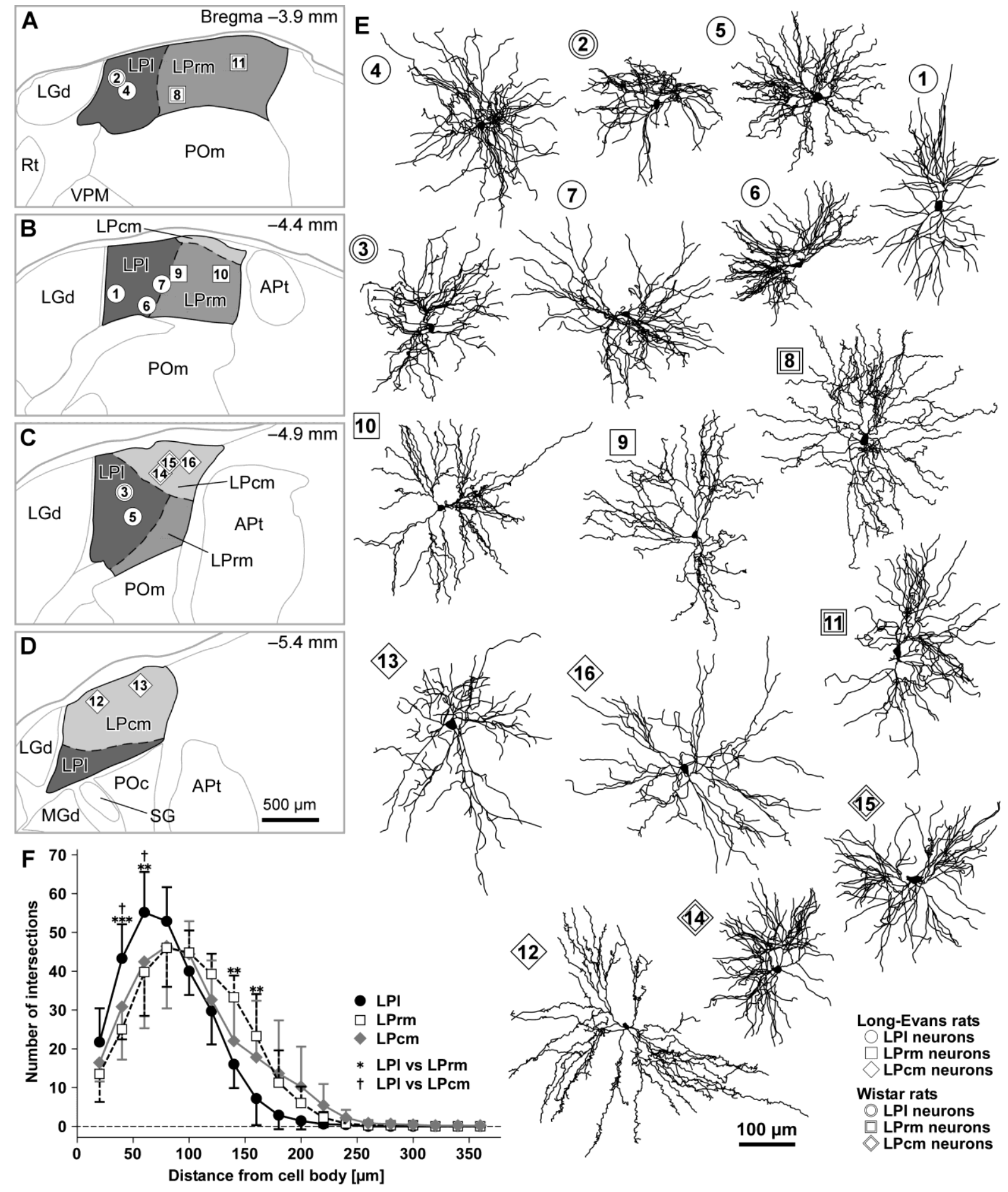

(5)
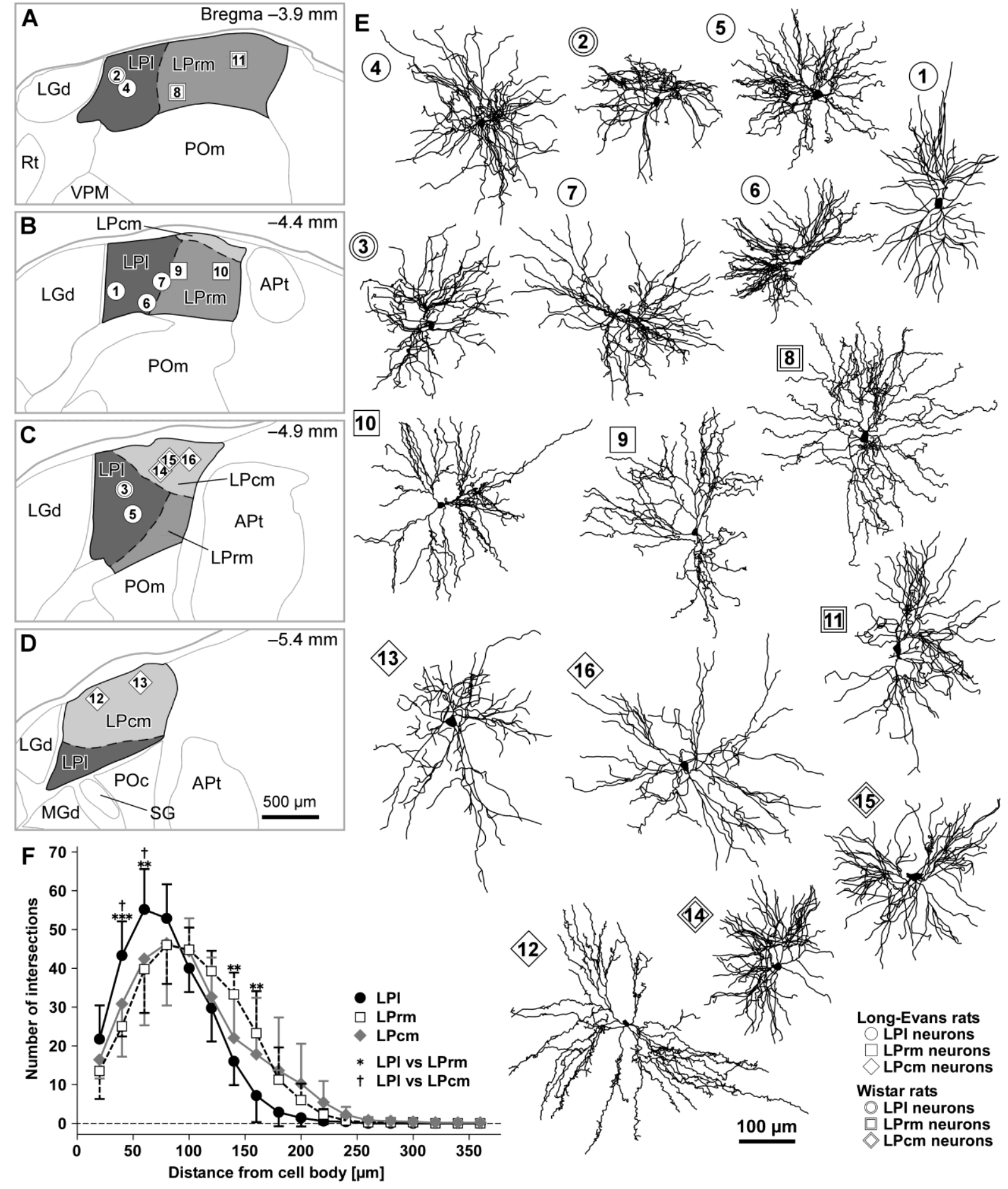

(6)

Figure 3

Figure 3. The locations and dendrites of reconstructed LP neurons. The locations of LP neurons were projected onto the nearest coronal plane (A-D), and these neurons serially numbered from the lateral to medial portions within the LPl (\#1-7), LPrm (\#8-11) and LPcm 
(\#12-16). The numbers surrounded with a single or double symbols (circle, square and diamond) indicate LP neurons of Long-Evans or Wistar rats, respectively. Dendrites are presented as projected figures onto the coronal plane $(\mathrm{E})$. The dendrites of neuron \#3, which were stained sagittally, were three-dimensionally reconstructed in software Neurolucida 10, and projected to the coronal plane. The dendritic fields of LP neuron subgroups were compared by Sholl analysis (F): black-filled circles, open squares, and grey-filled diamonds indicate the data of LPl, LPrm, and LPcm neurons, and the data are shown as mean \pm SD. Asterisks or daggers denote significant differences between LPl and LPrm neurons or between LPl and LPcm neurons $(\dagger p<0.05, * * p<0.01, * * * p<0.001$ by Bonferroni multiple comparison test). APt, anterior pretectal nucleus; MGd, dorsal division of the medial geniculate nucleus; POc, caudal sector of the posterior nucleus; SG, suprageniculate nucleus. Scale bar in D applies to A-D. 
A

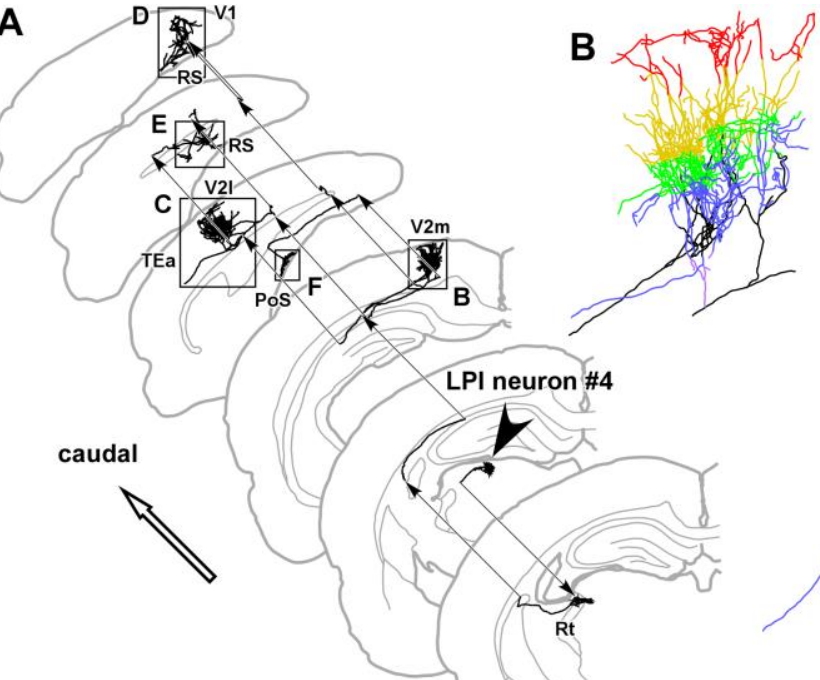

Fine varicose axon fibers:

in Layer I, red

in Layer II/III, yellow

in Layer IV, green

in Layer V, blue

in Layer VI, purple

Non-varicose axon fibers, black

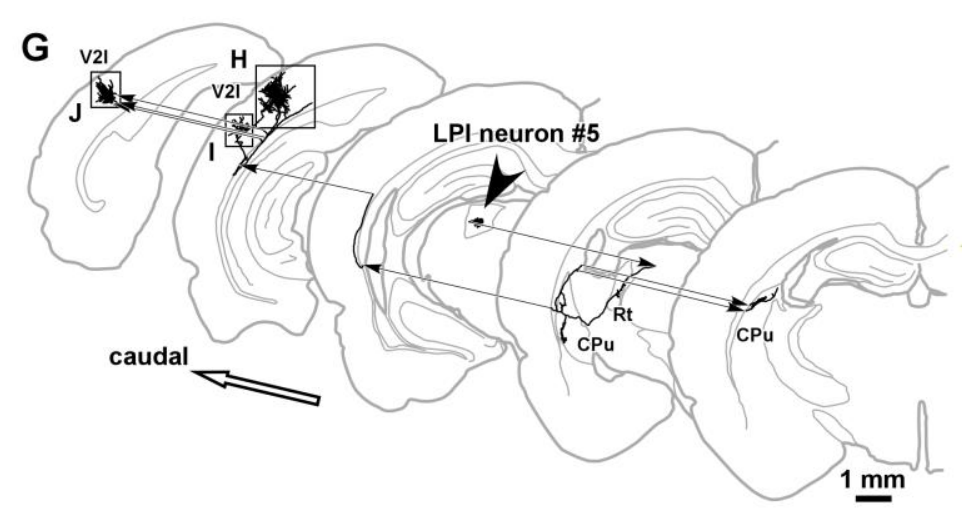

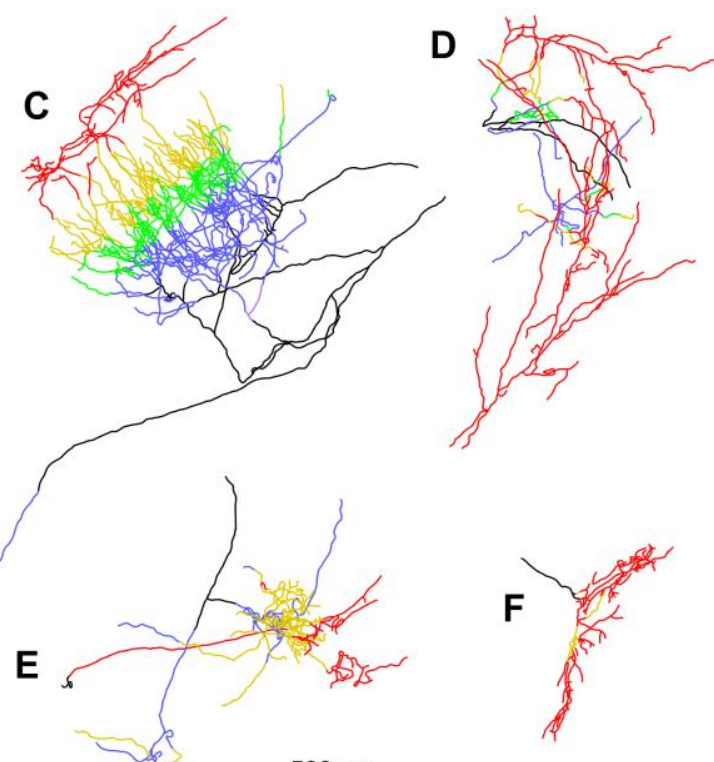

$500 \mu \mathrm{m}$
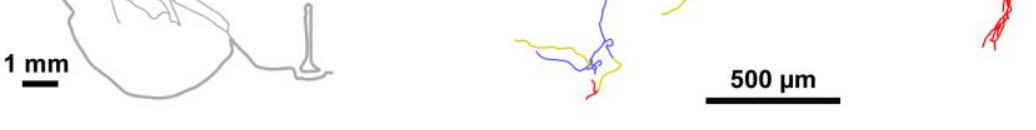

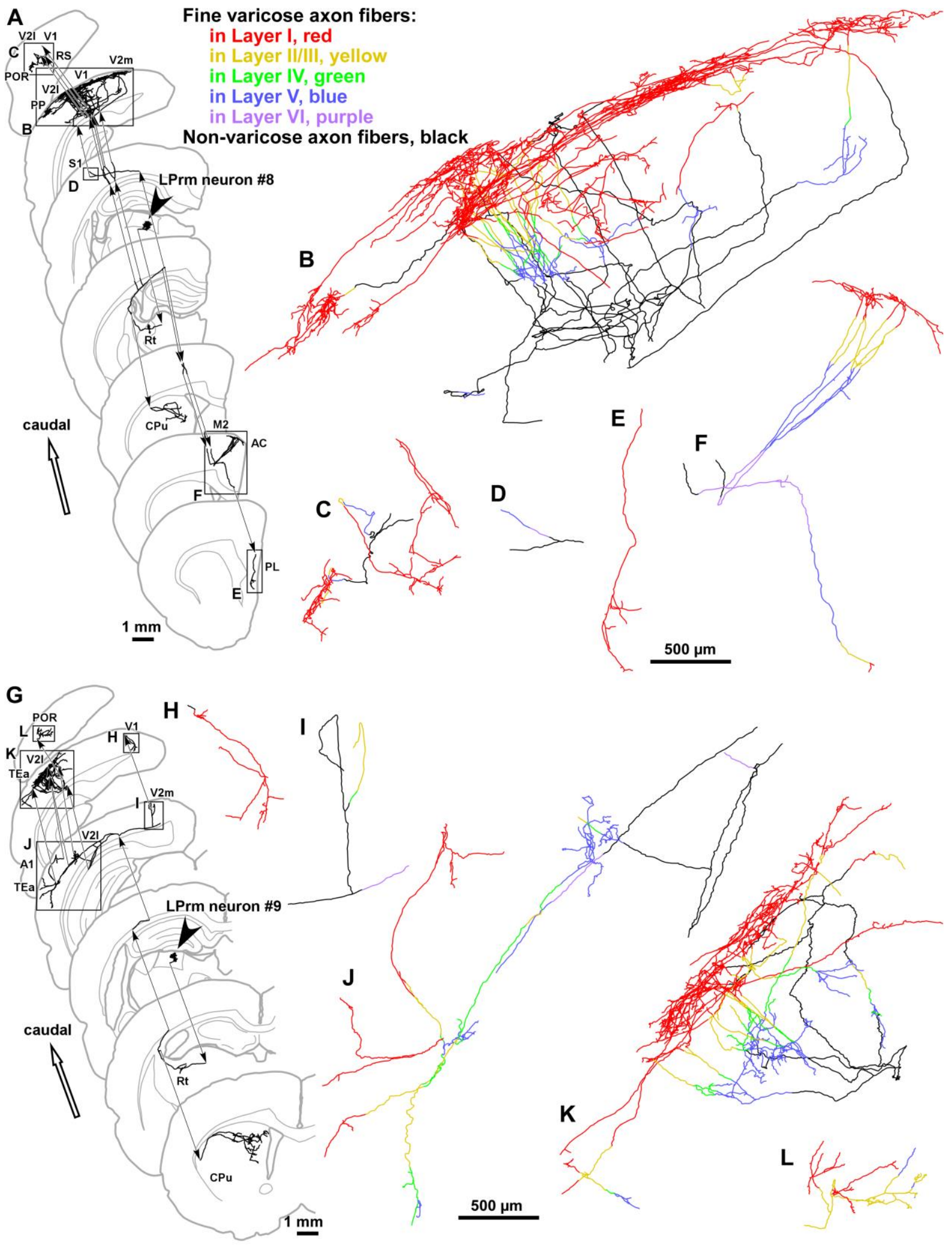

Figure 5

Figure 5. Axonal arborizations of LPrm neurons. The main targets of LPrm neurons were 
not only the V1 and V2 (A-C, G-K) but also the 'extravisual' areas such as the TEa (J, K).

Compared with LPl neurons, LPrm neurons sent many axon collaterals to L1 of the cortex (red lines in B, C, E, F, H, J-L). A1, primary auditory area; AC, anterior cingulate area; M2, secondary motor area; PL, prelimbic area; POR, postrhinal area; PP, posterior parietal area; S1, primary somatosensory area. 

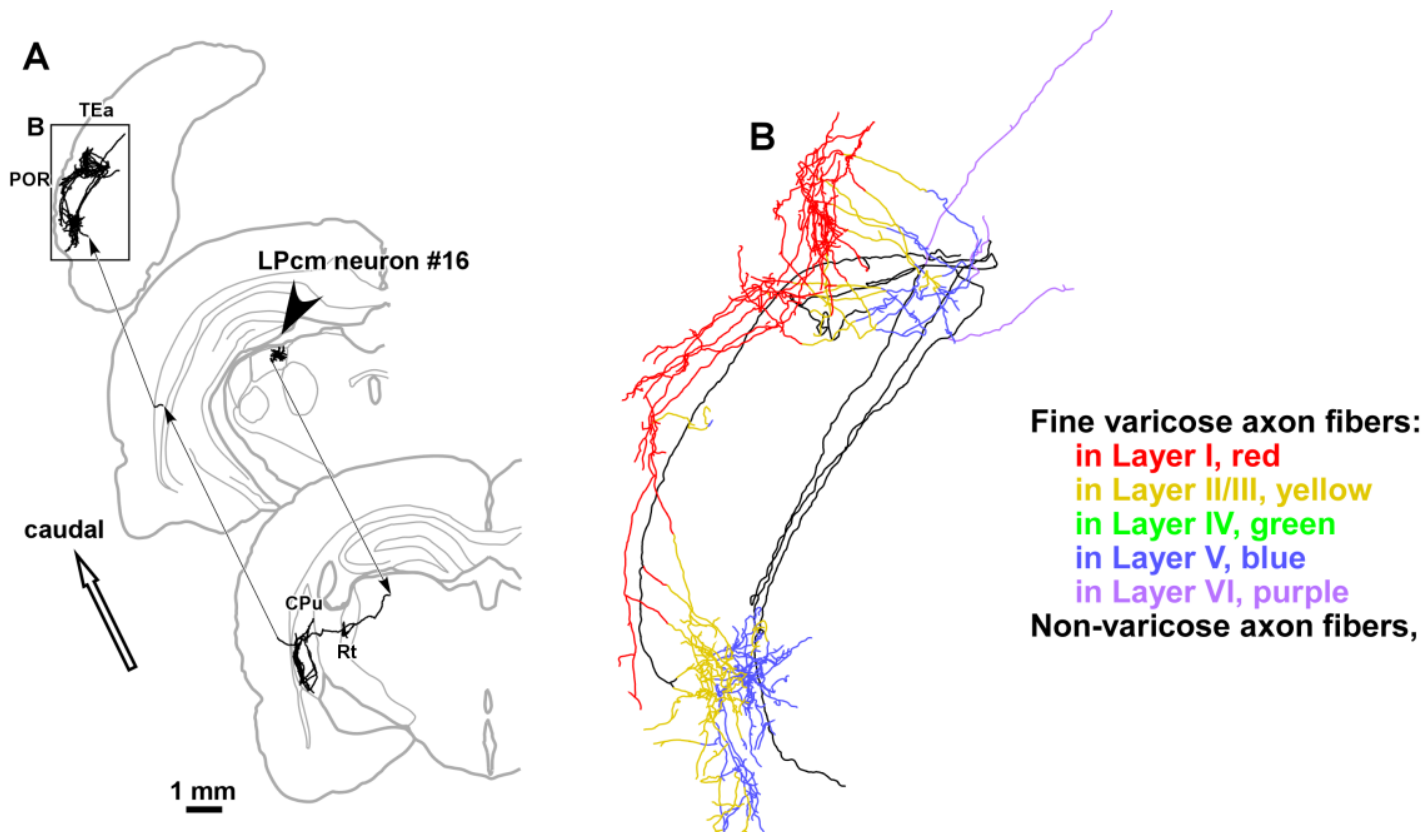

in Layer I, red

in Layer $\mathrm{V}$, blue

Non-varicose axon fibers, black
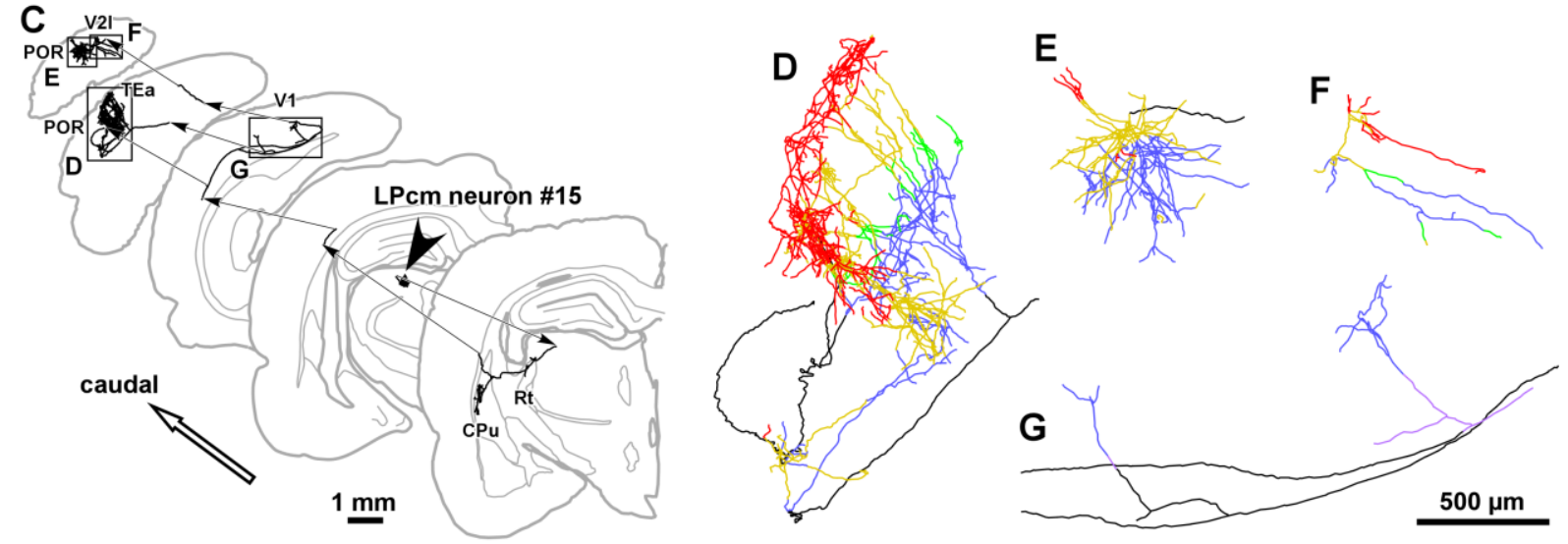

Figure 6

Figure 6. Axonal arborizations of LPcm neurons. The axonal fibers of LPcm neurons were mostly observed in the POR (A-E) and TEa (A-D), and found only sparsely in the V1 and V2 (F, G). 

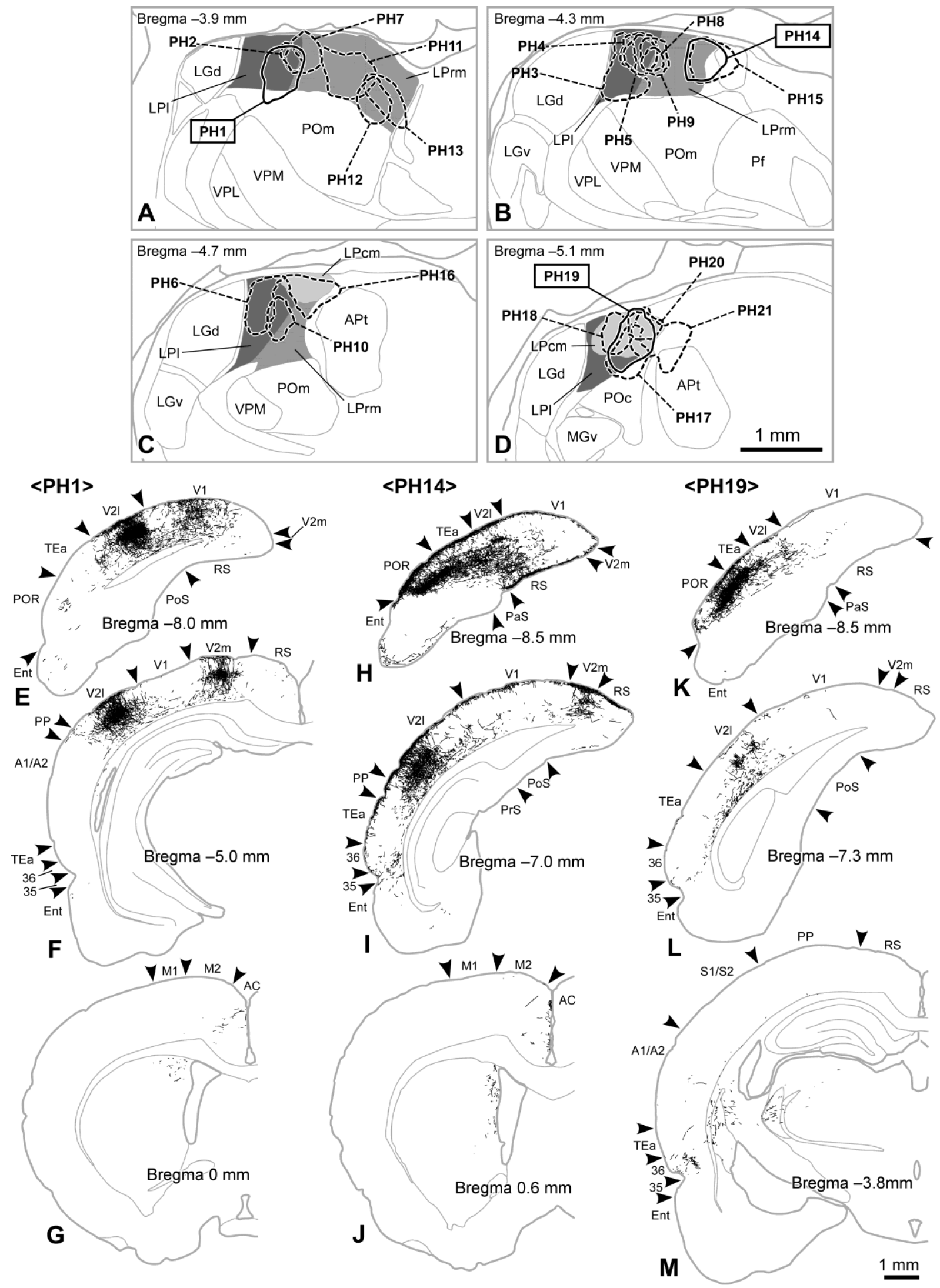

Figure 7 
Figure 7. Thalamocortical projection of LP neurons after injections of PHA-L into the three subdivisions of the LP. The injection sites of PHA-L were indicated on the drawing of the coronal planes $(\mathrm{A}-\mathrm{D})$. Three representative labeling in the cerebral cortex are shown $(\mathrm{E}-\mathrm{M})$. Ent, entorhinal area; 35, area 35; 36, area 36; A2, secondary auditory area; APt, anterior pretectal nucleus; Ent, entorhinal area; LGv, ventral lateral geniculate nucleus; M1, primary motor area; MGv, ventral division of the medial geniculate nucleus; PaS, parasubiculum; Pf, parafascicular nucleus; POc, caudal sector of the posterior nucleus; PrS, presubiculum; S1, primary somatosensory area; S2, secondary somatosensory area; VPL, ventral posterolateral nucleus. Scale bar in D applies to A-D; that in M to E-M. 

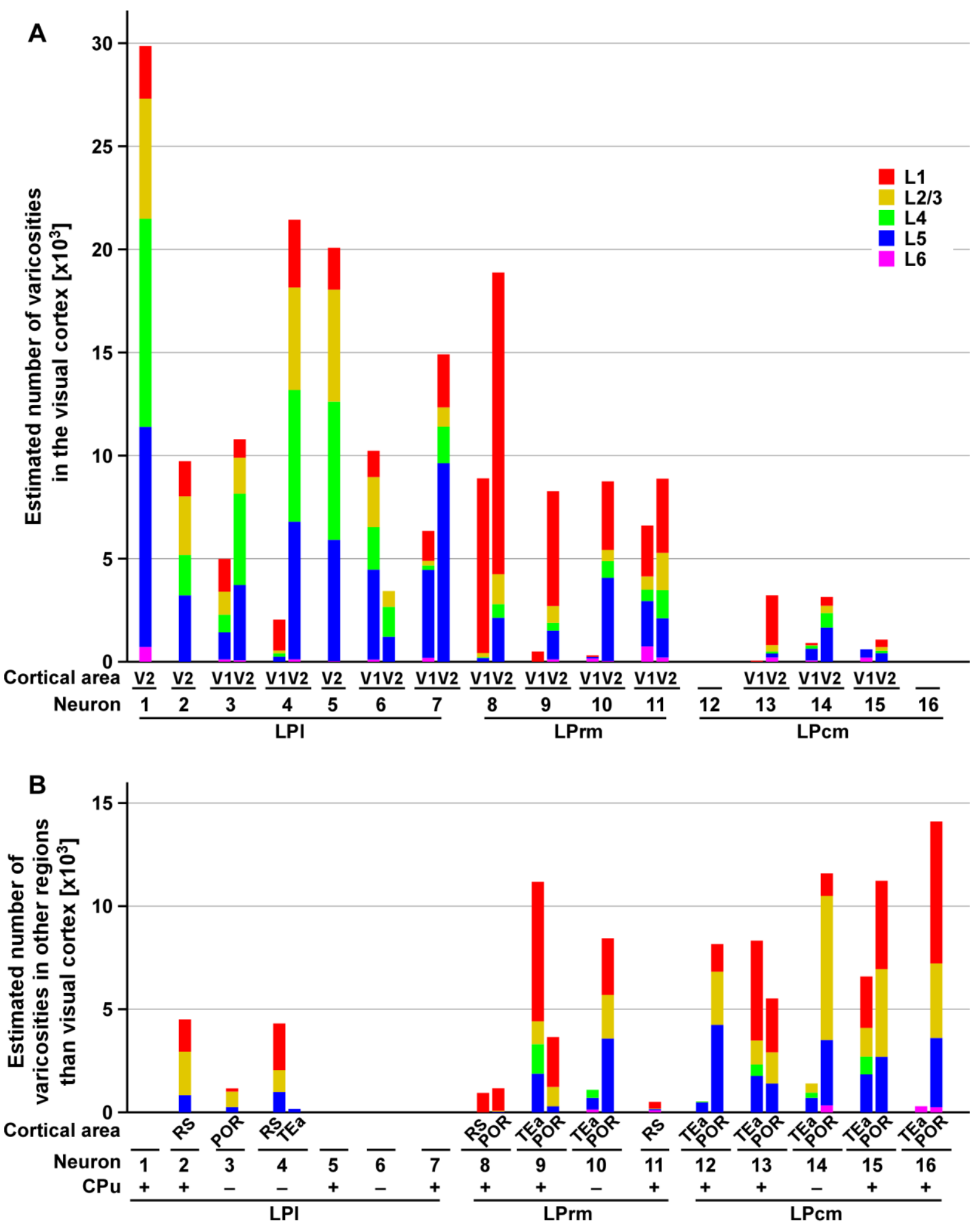

Figure 8

Figure 8. Laminar organization of thalamocortical axon varicosities of single LP neurons.

The estimated number of axonal varicosities in each cortical layer was calculated from the 
data on the axon length of the neurons and the density of axonal varicosities (Supplementary

Table 1). A: visual areas. B: 'extravisual areas', such as the retrosplenial (RS), postrhinal (POR) and temporal association areas (TEa). 

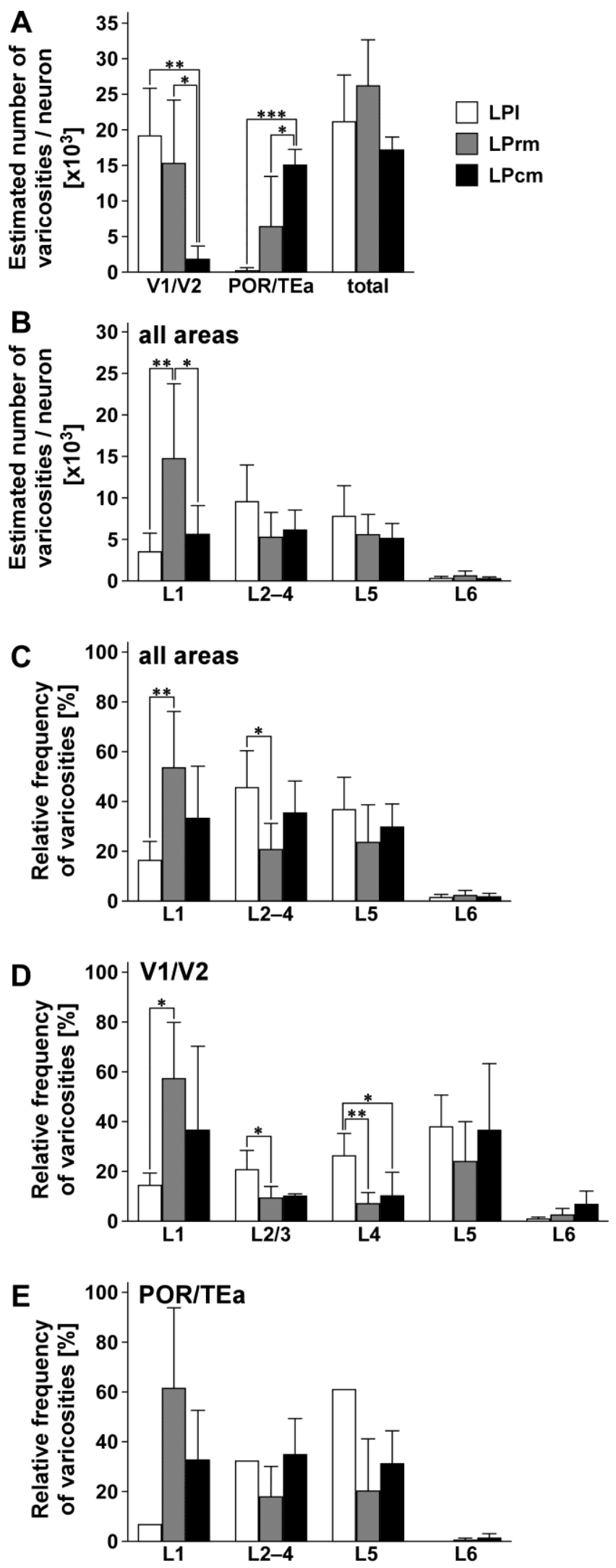

Figure 9

Figure 9. Statistical comparison of the estimated number of thalamocortical axon 
varicosities among three subdivisions of the LP. A: The number of varicosities in the visual areas $(\mathrm{V} 1, \mathrm{~V} 2)$ with that in the 'extravisual' postrhinal (POR) and temporal association areas (TEa). B: Laminar distribution of axon varicosities in the cerebral cortex. C: Relative laminar frequency of axon varicosities in the cerebral cortex. D: Relative laminar frequency of axon varicosities in the visual areas. $7 \mathrm{LPl}, 4 \mathrm{LPrm}$ and $3 \mathrm{LPcm}$ neurons were analyzed. E: Relative laminar frequency of axon varicosities in the POR and TEa. 2 LPl, 3 LPrm and $5 \mathrm{LPcm}$ neurons were examined. Each bar and error bar represents the mean and SD, respectively. $* \mathrm{p}<0.05, * * \mathrm{p}<0.01, * * * \mathrm{p}<0.001$ by Tukey's post hoc multiple comparison test following one-way ANOVA. 


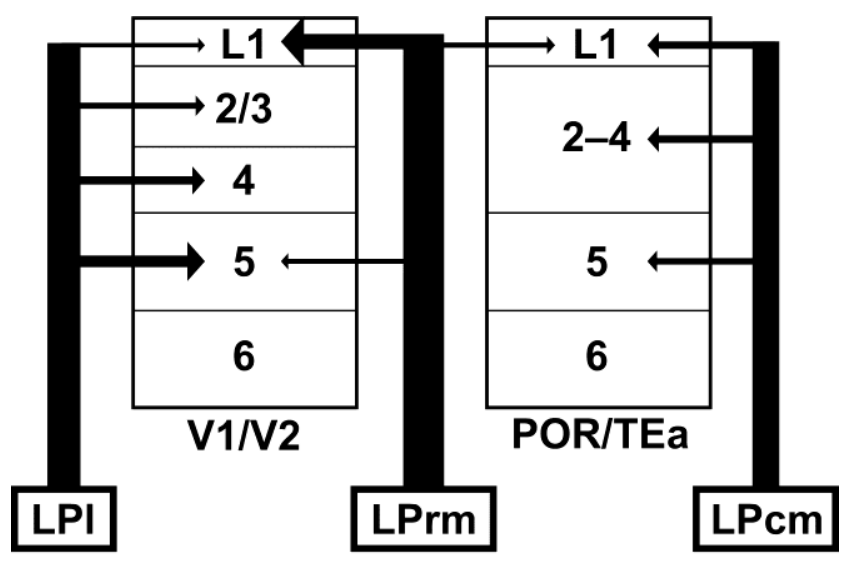

Figure 10

Figure 10. Schematic diagram of LP-cortical projections. Line thickness and arrowhead size are proportional to the mean estimated number of varicosities. The largest and smallest numbers illustrated as arrows in the figure are 26178 varicosities/neuron (total projections of LPrm neurons) and 2696 varicosities/neuron (projections of LP1 neurons to L1 of the V1/V2), respectively. Projections with $\leq 1585$ varicosities/neuron are not depicted here. POR, postrhinal area; TEa, temporal association area. 


\title{
Supporting Information
}

\section{Different Cortical Projections from Three Subdivisions of the Rat Lateral Posterior Thalamic Nucleus: A Single Neuron Tracing Study with Viral Vectors}

\author{
Hisashi Nakamura, Hiroyuki Hioki*, Takahiro Furuta and Takeshi Kaneko
}

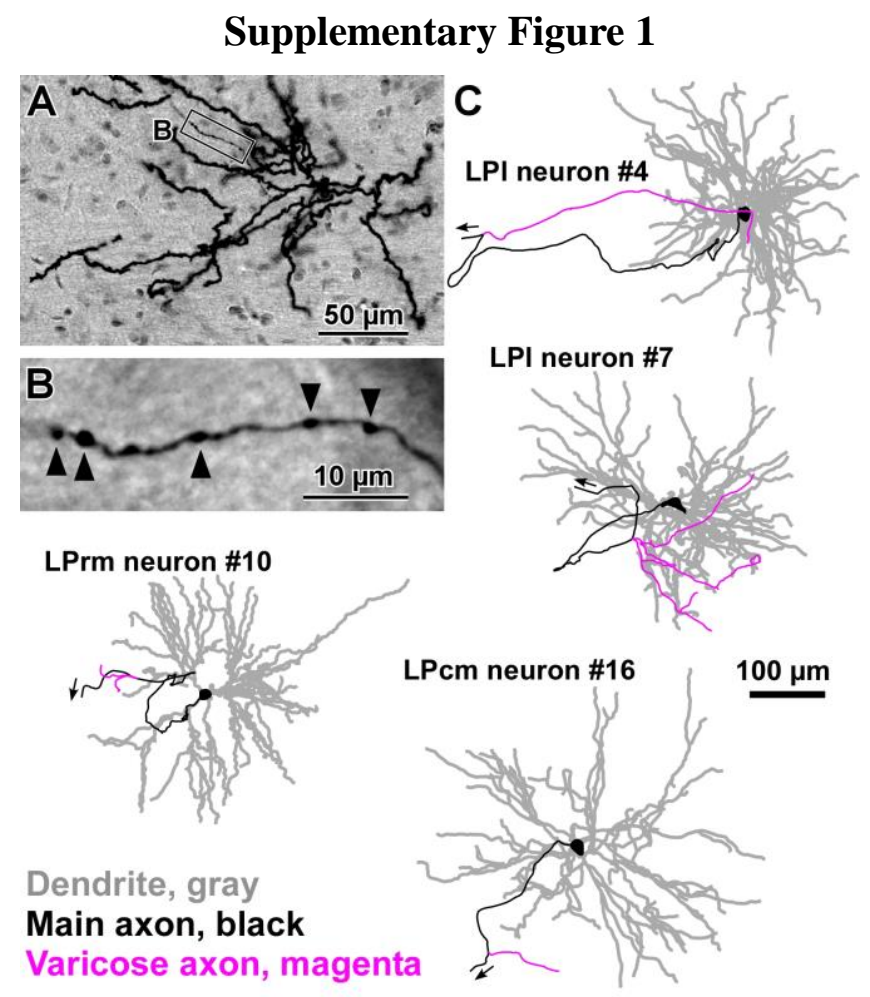

\section{Supplementary Figure 1.}

Intrathalamic axon collaterals. A: Cell body and dendrites of neuron \#7. B: Varicose axon collateral. Arrowheads indicate the axon varicosities which were located at a focal plane of the microscope. C: Four of 16 reconstructed LP neurons, 2 LPl (\#4 and \#7), 1 LPrm (\#10) and 1 LPcm (\#16) neurons, emitted local axon collaterals (magenta lines). It is noticed that all the collaterals were poorly developed. 


\section{Supplementary Figure 2}
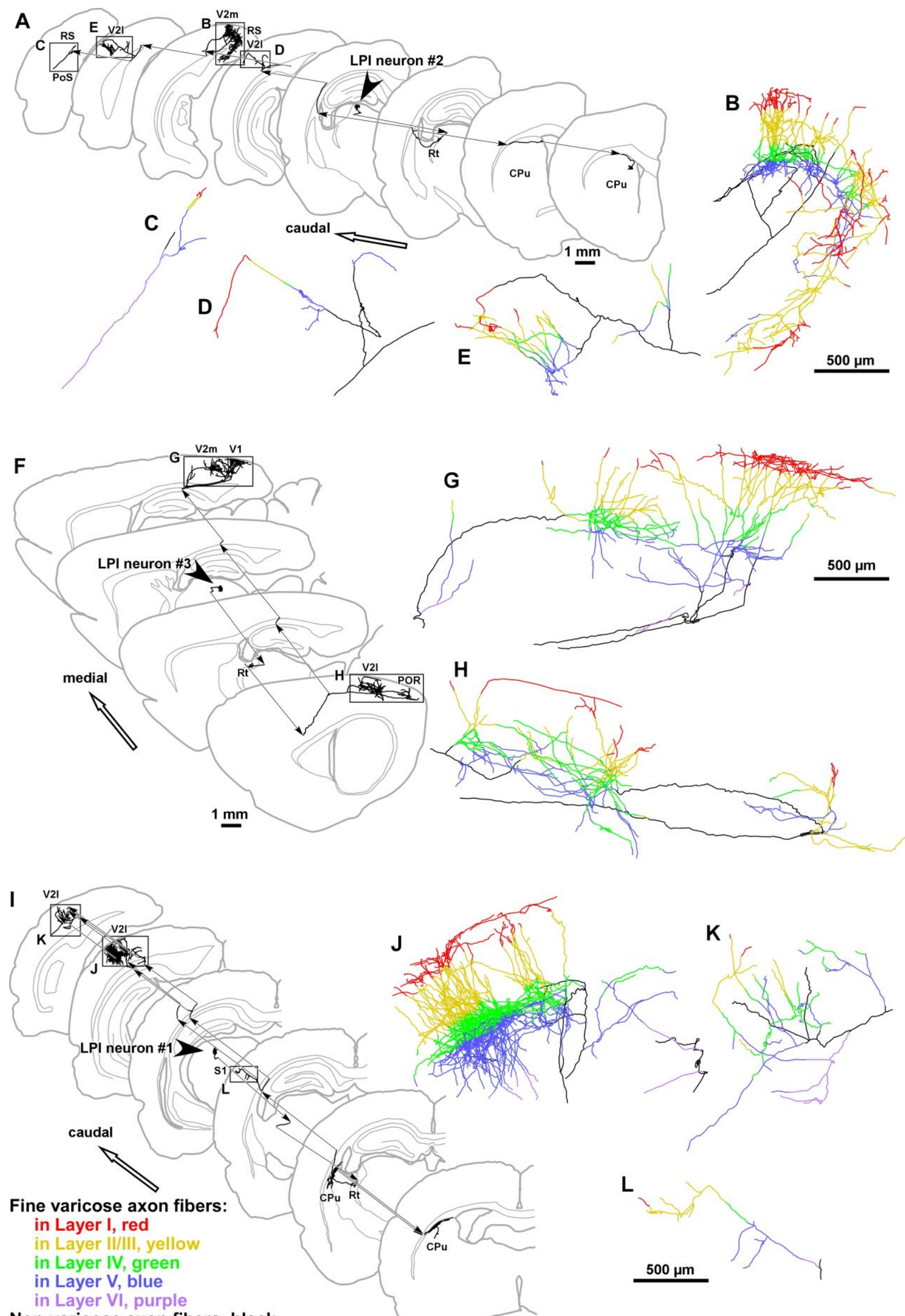

Non-varicose axon fibers, black
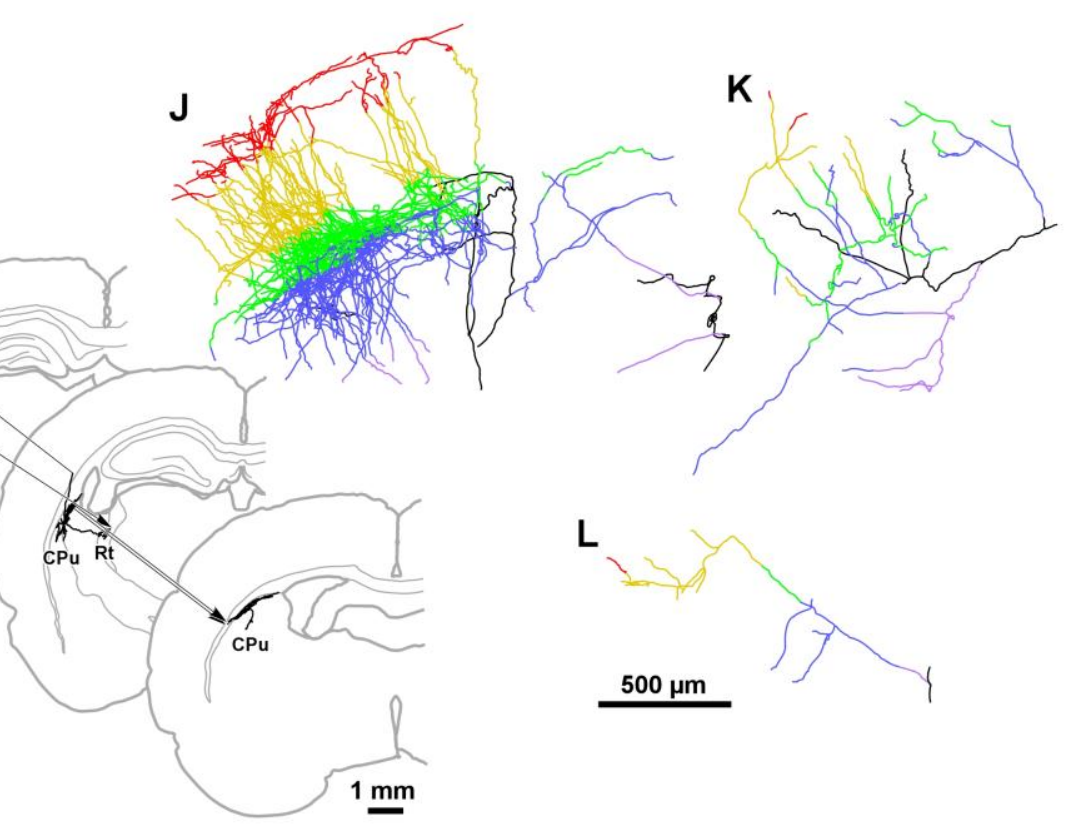

L

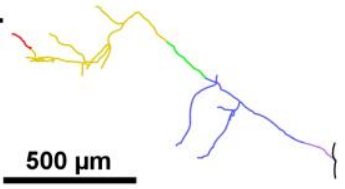




\section{Supplementary Figure 2.}

Axonal arborizations of LP1 neurons \#1-3. LP1 neurons in coronal (\#1 and \#2) or sagittal (\#3) sections sent their axons mainly to the secondary visual area (V2; A, F, I). These neurons formed at least two axonal clusters in the V2 (B, E, G, H, J, K). Some of the LP1 neurons also extended axons to the primary visual $(\mathrm{V} 1 ; \mathrm{G})$, retrosplenial $(\mathrm{RS} ; \mathrm{B}, \mathrm{C})$, postrhinal (POR; H), postsubicular $(\mathrm{PoS} ; \mathrm{C})$ and primary somatosensory areas $(\mathrm{S} 1 ; \mathrm{L}) . \mathrm{CPu}$, caudate-putamen; Rt, thalamic reticular nucleus; V21, lateral region of the $\mathrm{V} 2 ; \mathrm{V} 2 \mathrm{~m}$, medial region of the $\mathrm{V} 2$. 


\section{Supplementary Figure 3}

Fine varicose axon fibers:

in Layer I, red

in Layer II/III, yellow

in Layer IV, green

in Layer $\mathrm{V}$, blue

in Layer VI, purple

Non-varicose axon fibers, black

B
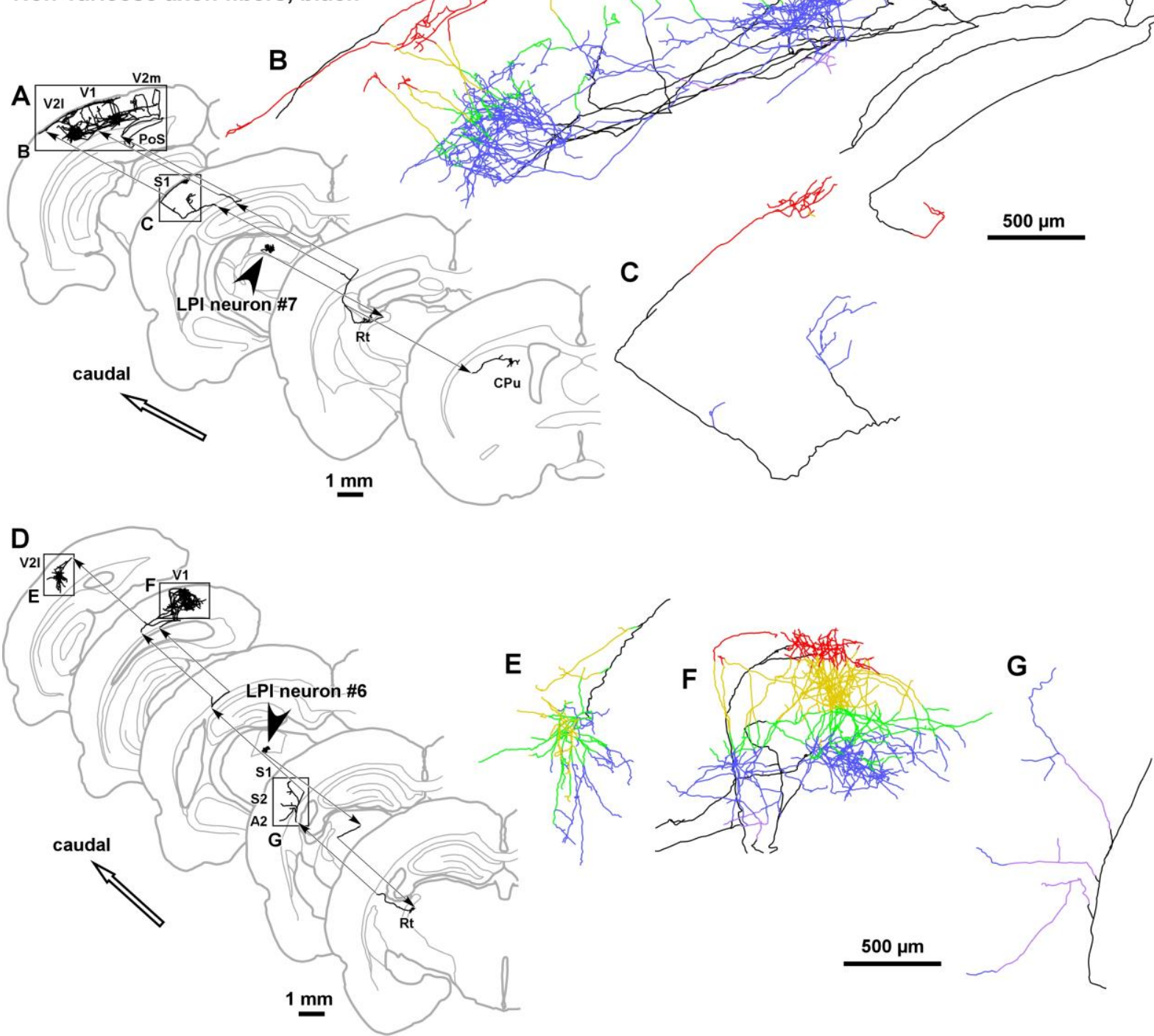

\section{Supplementary Figure 3.}

Axonal arborizations of LPl neurons \#6 and \#7. These LPl neurons sent their axons densely to the visual areas (A, D). The two neurons formed axonal clusters in the V1 (B, F) as well as in the V2 (B, E). They also sent several axon collaterals to the PoS (B), S1 (C, G), secondary somatosensory (S2; G) and secondary auditory areas (A2; G). For abbreviations, see the legend of Supplementary Figure 2. 


\section{Supplementary Figure 4}
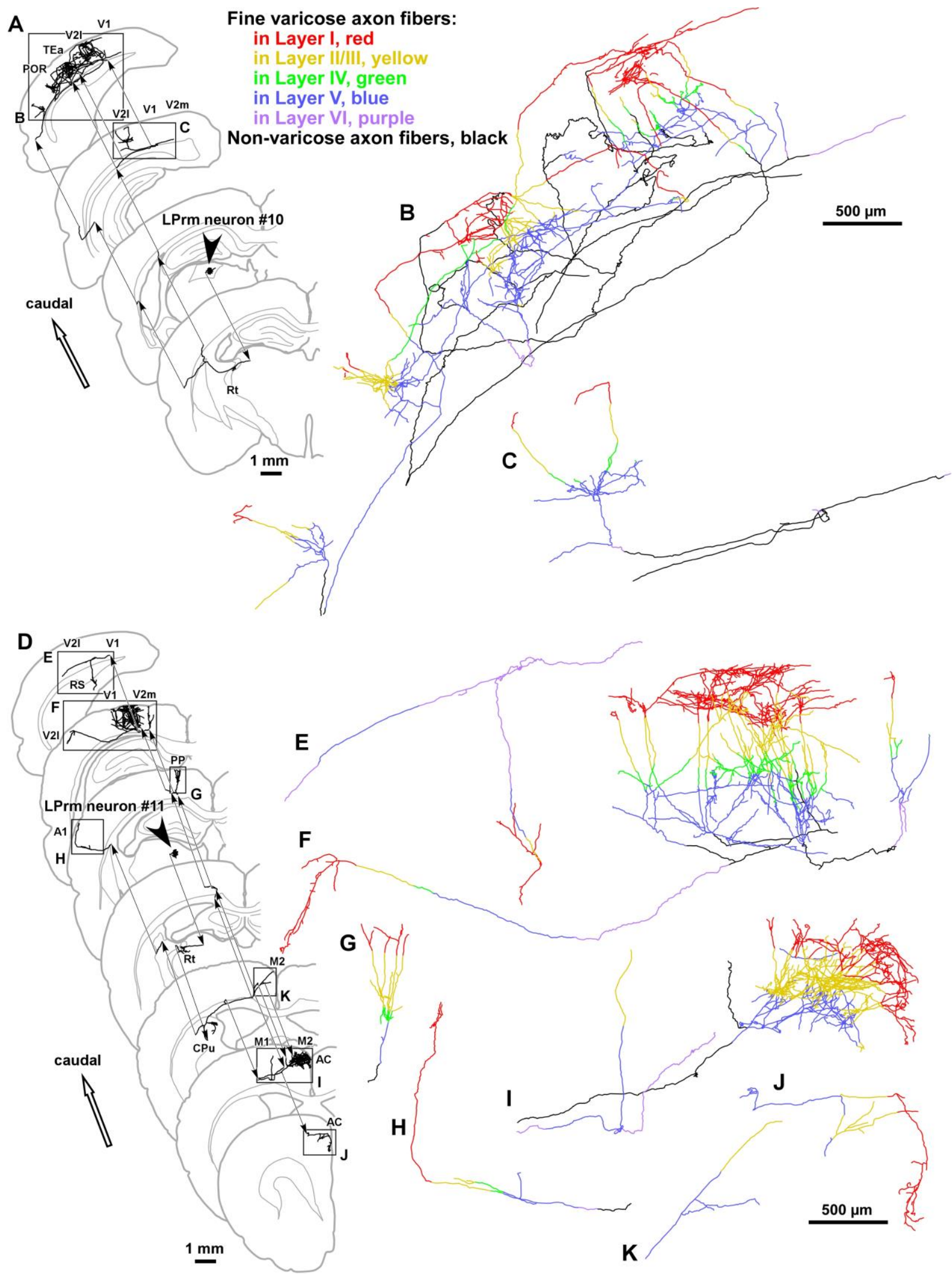


\section{Supplementary Figure 4.}

Axonal arborizations of LPrm neurons \#10 and \#11. The main targets of these LPrm neurons were the V1 (A-F), V2 (A-F), POR (A, B), secondary motor (M2; D, K, I) and anterior cingulate areas $(\mathrm{AC} ; \mathrm{D}, \mathrm{I}, \mathrm{J})$. Their axons were also observed in the RS (E), temporal association $(\mathrm{TEa} ; \mathrm{B})$, posterior parietal $(\mathrm{PP} ; \mathrm{G})$, primary auditory $(\mathrm{A} 1 ; \mathrm{H})$ and primary motor areas (M1; I). For abbreviations, see the legend of Supplementary Figure 2. 


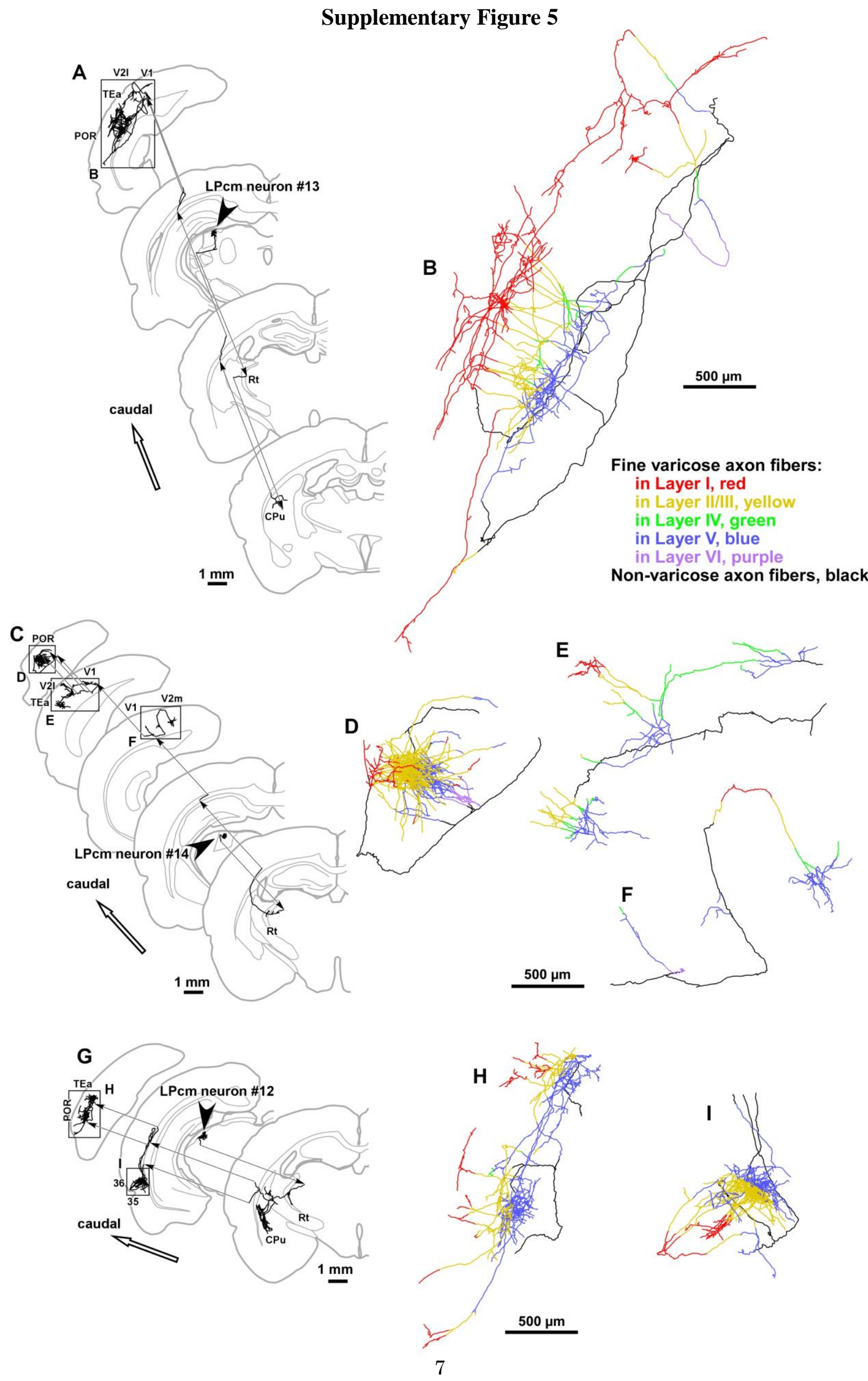




\section{Supplementary Figure 5.}

Axonal arborizations of LPcm neurons \#12-14. These LPcm neurons formed dense axonal plexus in areas 35 and 36 (G, I) and the TEa (A-C, E, G, H) and POR (A-D, G, H). In the V1 and $\mathrm{V} 2$, on the other hand, only sparse axons of these neurons were observed $(\mathrm{B}, \mathrm{E}, \mathrm{F})$. For abbreviations, see the legends of Supplementary Figures 2 and 4. 
Supplementary Table 1. Inter-varicosity interval of axon fibers from LP neurons in each cortical area and layer.

\begin{tabular}{lccccc}
\hline cortex \layer & L1 & L2/3 & L4 & L5 & L6 \\
\hline V1 & $7.7 \pm 4.41$ & $10.22 \pm 5.93$ & $6.87 \pm 3.72$ & $7.21 \pm 4.5$ & $7.84 \pm 5.3$ \\
V2 & $4.74 \pm 2.71$ & $6.73 \pm 3.48$ & $5.14 \pm 2.71$ & $5.69 \pm 3.8$ & $7.25 \pm 4.39$ \\
RS & $6.31 \pm 3.73$ & $10.13 \pm 6.21$ & - & $8.56 \pm 8.88$ & $9.27 \pm 7.43$ \\
TEa & $4.17 \pm 2.24$ & $6.69 \pm 3.79$ & $4.69 \pm 2.67$ & $6.88 \pm 4.19$ & $6.75 \pm 4.39$ \\
POR & $4.23 \pm 2.43$ & $6.33 \pm 3.9$ & - & $6.39 \pm 3.87$ & $6.44 \pm 3.69$ \\
$35 / 36$ & $4.35 \pm 2.04$ & $7.05 \pm 3.98$ & - & $6.66 \pm 3.93$ & $5.33 \pm 3.08$ \\
PoS & $7.81 \pm 5.46$ & $9.87 \pm 6.67$ & - & $11.11 \pm 7.08$ & $9.62 \pm 5.9$ \\
S1/S2 & $6.97 \pm 3.95$ & $10.25 \pm 6.87$ & $7.05 \pm 4.14$ & $6.44 \pm 4.53$ & $8.31 \pm 5.75$ \\
PP & $6.21 \pm 3.52$ & $8.53 \pm 6.31$ & $8.29 \pm 5.02$ & $8.81 \pm 6.15$ & $8.56 \pm 6.75$ \\
A1/A2 & $6.48 \pm 4.56$ & $7.62 \pm 5.13$ & $6.14 \pm 3.38$ & $8.1 \pm 4.81$ & $6.76 \pm 5.48$ \\
M1/M2 & $6.05 \pm 3.75$ & $6.99 \pm 4.16$ & - & $8.09 \pm 5.75$ & $5.45 \pm 4.84$ \\
PL & $7.38 \pm 4.38$ & $11.1 \pm 6.12$ & - & $9.46 \pm 7.31$ & $10.26 \pm 6.15$ \\
AC & $7.38 \pm 4.41$ & $8.39 \pm 5.82$ & - & $7.63 \pm 5.4$ & $9.25 \pm 7.37$ \\
\hline
\end{tabular}

The intervals (mean \pm S.D. in $\mu \mathrm{m}, \mathrm{n}=100$ ) were measured at the axonal fibers that were anterogradely labeled after injections of PHA-L into the LP and ran parallel to the section surface. 35, area 35; 36, area 36; PL, prelimbic area; for other abbreviations, see the legends of Supplementary Figures 2-5. 